\title{
A cytotoxic anti-IL-3R $\alpha$ antibody targets key cells and cytokines implicated in systemic lupus erythematosus
}

\author{
Shereen Oon, ${ }^{1,2,3}$ Huy Huynh, ${ }^{4}$ Tsin Yee Tai, ${ }^{4}$ Milica Ng, ${ }^{4}$ Katherine Monaghan, ${ }^{4}$ Mark Biondo, ${ }^{4}$ \\ Gino Vairo, ${ }^{4}$ Eugene Maraskovsky, ${ }^{4}$ Andrew D. Nash, ${ }^{4}$ Ian P. Wicks, $, 1,2,3$ and Nicholas J. Wilson ${ }^{4}$ \\ 'Division of Inflammation, The Walter and Eliza Hall Institute of Medical Research, Parkville, Victoria, Australia. \\ ${ }^{2}$ Department of Rheumatology, The Royal Melbourne Hospital, Parkville, Victoria, Australia. \\ ${ }^{3}$ The University of Melbourne, Parkville, Victoria, Australia. ${ }^{4}$ CSL Limited, Parkville, Victoria, Australia.
}

To date, the major target of biologic therapeutics in systemic lupus erythematosus (SLE) has been the B cell, which produces pathogenic autoantibodies. Recently, targeting type I IFN, which is elaborated by plasmacytoid dendritic cells (pDCs) in response to endosomal TLR7 and TLR9 stimulation by SLE immune complexes, has shown promising results. pDCs express high levels of the IL-3R $\alpha$ chain (CD123), suggesting an alternative potential targeting strategy. We have developed an anti-CD123 monoclonal antibody, CSL362, and show here that it affects key cell types and cytokines that contribute to SLE. CSL362 potently depletes pDCs via antibody-dependent cellmediated cytotoxicity, markedly reducing TLR7, TLR9, and SLE serum-induced IFN- $\alpha$ production and IFN- $\alpha$-upregulated gene expression. The antibody also inhibits TLR7- and TLR9-induced plasmablast expansion by reducing IFN- $\alpha$ and IL-6 production. These effects are more pronounced than with IFN- $\alpha$ blockade alone, possibly because pDC depletion reduces production of other IFN subtypes, such as type III, as well as non-IFN proinflammatory cytokines, such as IL-6. In addition, CSL362 depletes basophils and inhibits IL-3 signaling. These effects were confirmed in cells derived from a heterogeneous population of SLE donors, various IFN-dependent autoimmune diseases, and healthy controls. We also demonstrate in vivo activity of CSL362 following its s.c. administration to cynomolgus monkeys. This spectrum of effects provides a preclinical rationale for the therapeutic evaluation of CSL362 in SLE.

Authorship note: I.P. Wicks and N.J. Wilson contributed equally to this work.

Conflict of interest: S. Oon and I.P. Wicks have received funding from CSL Limited. H. Huynh, TY. Tai, M. Ng, K. Monaghan, M. Biondo, C. Vairo, E. Maraskovsky, A.D. Nash, and N.J. Wilson are employees of CSL Limited. K. Monaghan, G. Vairo, E. Maraskovsky, A.D. Nash, and N.J. Wilson hold stock in CSL Limited.

Submitted: December 21, 2015 Accepted: April 1, 2016 Published: May 5, 2016

Reference information: JCI Insight. 2016;1(6):e86131. doi:10.1172/jici.insight.86131.

\section{Introduction}

Systemic lupus erythematosus (SLE) is a multisystem autoimmune disease, with significant morbidity and increased mortality $(1,2)$, in part because of current treatment limitations. Given the importance of autoantibodies in the pathogenesis of SLE, many current biologic therapies, such as rituximab and belimumab, target B cells. A wealth of data, including the peripheral blood IFN gene "signature" (3) and elevated type I IFN and IFN-regulated chemokines in SLE sera (4), also supports a central role for type I IFN in SLE. Importantly, recent clinical trials with monoclonal antibodies (mAbs) targeting IFN- $\alpha$ (5-7) and the type I IFN receptor (IFNAR) (8) have demonstrated reductions in the IFN gene signature and disease activity measures.

Plasmacytoid dendritic cells (pDCs) are specialized dendritic cells and are the major producers of type I IFNs (9) following endosomal TLR7 and TLR9 activation by pathogen-associated molecular patterns and human-derived nucleic acids (10). In SLE, immune complexes containing host-derived nucleic acids and a variety of autoantibodies stimulate TLR7 and TLR9 in pDCs to promote IFN production (11-16). Recently, murine models of lupus provided direct evidence for the pathogenic role of pDCs $(17,18)$. In contrast, evidence implicating pDCs in human SLE has been indirect, with reports of altered circulating pDC numbers (19-22), abundant pDCs producing IFN- $\alpha / \beta$ in cutaneous lupus $(19,23)$, and TLR9-mediated $\mathrm{pDC}$ activation by DNA-containing immune complexes in vitro $(15,24)$. In contrast to B cells, therapeutic targeting of pDCs in SLE is still in its infancy (25-27).

pDCs highly express IL-3R $\alpha$ (CD123) compared with other peripheral blood cells $(23,28)$. CSL362 is a humanized therapeutic mAb that binds to CD123 and incorporates two mechanisms of action. It inhibits IL-3 binding to CD123, antagonizing IL-3 signaling in target cells $(29,30)$. Second, the Fc region of CSL362 has been mutated to increase affinity for CD16 (FcyRIIIa), thereby enhancing antibody- 
dependent cell-mediated cytotoxicity (ADCC). CSL362 can induce ADCC against CD123+ acute myeloid leukemia (AML) blasts and leukemic stem cells in vitro and reduces leukemic cell growth in murine xenograft models of human AML (30). A phase I clinical trial of CSL362 in AML has recently completed (clinical trial NCT01632852).

In this study, we explored the potential utility of CSL362 in primary human cells derived from patients with SLE. We found that CSL362 potently depleted pDCs and inhibited TLR7- and TLR9-stimulated IFN- $\alpha$ production and IFN- $\alpha$-inducible gene expression ex vivo in SLE patients. This effect was confirmed in vivo, with s.c. administration of CSL362 in cynomolgus monkeys. Basophils, which also express high levels of CD123 and are thought to contribute to the pathology of SLE (31), were likewise depleted. In addition, CSL362 inhibited pDC-dependent plasmablast expansion ex vivo. These findings demonstrate that, through targeting IL-3R $\alpha$, CSL362 directly and indirectly affects key cells contributing to SLE and provide a preclinical rationale for CSL362's evaluation in this complex disease, for which more therapeutic options are urgently required.

\section{Results}

pDCs and basophils have high CD123 expression and are selectively depleted by CSL362. Cell surface expression of CD123 was examined on peripheral blood cells from a heterogeneous cohort of SLE donors $(n=34)$ (Supplemental Table 1; supplemental material available online with this article; doi:10.1172/jci.insight.86131DS1), autoimmune disease control donors $(n=20)$, and healthy control donors $(n=34)$. Of the cell subsets evaluated, pDCs and basophils had the highest CD123 expression ( 40,000 and 20,000 receptors/cell, respectively; Figure 1A), with expression being highest on pDCs in most donors. Expression in all other cell types was much lower and ranged from approximately 2,000 receptors/cell in myeloid dendritic cells (mDCs) and intermediate monocytes to less than approximately 1,000 receptors/cell in the other cell types.

Given the selective, high-level CD123 expression on pDCs and basophils, we evaluated the ability of the anti-CD123 mAb, CSL362, to deplete these cell types. An isotype control mAb (isotype control) and Fab'CSL362 (the Fab fragment of CSL362 that lacks the Fc portion but retains IL-3-neutralizing activity) were used as comparisons. At 24 hours, pDCs were potently and reproducibly depleted by CSL362 but not the isotype control or Fab'CSL362 (Figure 1, B and C), demonstrating that ADCC, and not IL-3 neutralization, is the main mechanism of depletion. Basophils were less completely depleted than pDCs (Figure $1, \mathrm{~B}$ and D), possibly due to higher circulating numbers and relatively lower CD123 expression. A higher dose of Fab'CSL362 also depleted pDCs but not basophils (Supplemental Figure 1, A and B), reflecting the requirement of IL-3 for pDC survival (32). Depletion by CSL362 was selective for pDCs and basophils, as cells that express lower CD123 levels (mDCs, monocytes, and NK, B, and T cell subsets) were not depleted (Supplemental Figure 2, A-I).

CSL362 has been engineered for enhanced ADCC through mutations in its Fc region that increase its affinity for Fc $\gamma$ RIII (also known as CD16) expressed on NK cells (30). We therefore examined the effect of CSL362 on NK cell activation (percentage of CD107a+ NK cells). Fab'CSL362 and the isotype control, and a second isotype control (isotype 2) with an unmodified IgG1-Fc, were used for comparison. CSL362 and isotype 1, but not Fab'CSL362 or isotype 2, activated NK cells in SLE, autoimmune, and healthy control donors (Figure 1E), confirming that the modified IgG1-Fc mediates NK cell activation.

Depletion of pDCs by CSL362 inhibits TLR7, TLR9, and SLE serum-stimulated IFN- $\alpha$ production and IFN- $\alpha$ inducible gene expression. Given the potent depletion of pDCs by CSL362, we next evaluated its effect on TLR-stimulated IFN- $\alpha$ production and IFN-inducible gene expression. Pretreatment with CSL362 inhibited TLR7-stimulated (imiquimod) and TLR9-stimulated (CpG C) IFN- $\alpha$ production in both SLE donors and healthy donors (Figure 2A), whereas TLR4-stimulated (LPS) production was not significantly reduced by CSL362. There was robust induction of an IFN gene "signature," comprising 11 previously described IFN-inducible genes (3) (IFI44L, IFIT1, IFIT3, IRF7, ISG15, MX1, MX2, OAS1, OAS2, SERPING1, and $X A F 1$ ) in healthy donors after TLR3, TLR4, TLR7, TLR8, and TLR9 stimulation but weak induction by TLR5 (Figure 2B). The TLR7- and TLR9-stimulated IFN gene signature was completely and selectively neutralized by CSL362. As CSL362 specifically depletes pDCs and basophils, and pDCs selectively express TLR7 and TLR9 (33), these data suggest that the decrease in IFN- $\alpha$ production and gene expression is a direct effect of $\mathrm{pDC}$ depletion. We confirmed this by removing $\mathrm{pDCs}$ or basophils from peripheral blood mononuclear cells (PBMCs) by FACS sorting. Removal of pDCs, but not basophils, markedly reduced TLR7- and TLR9-induced IFN- $\alpha$ production (Figure 2C). These data imply that other cell types are unable 
A

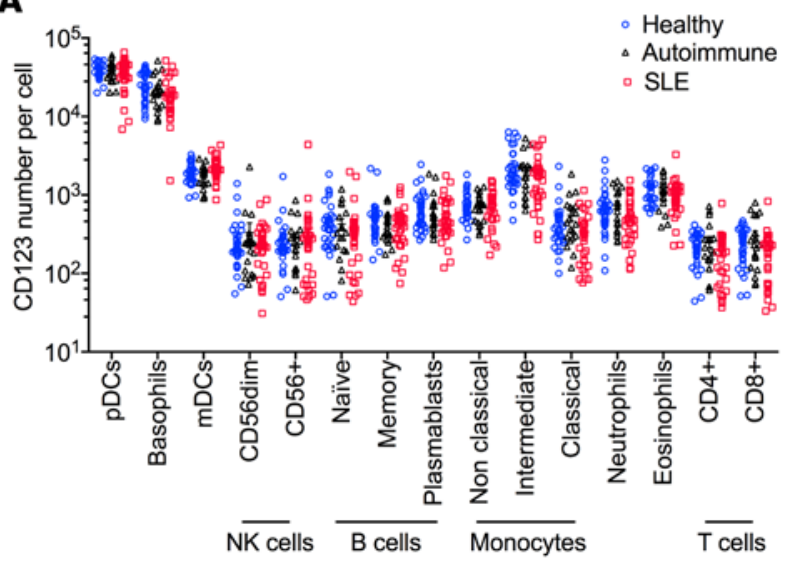

B
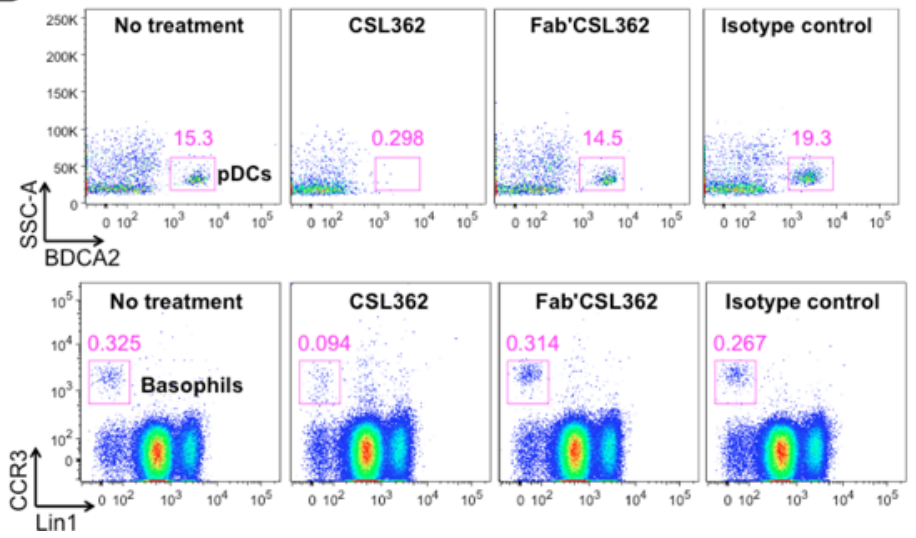

C

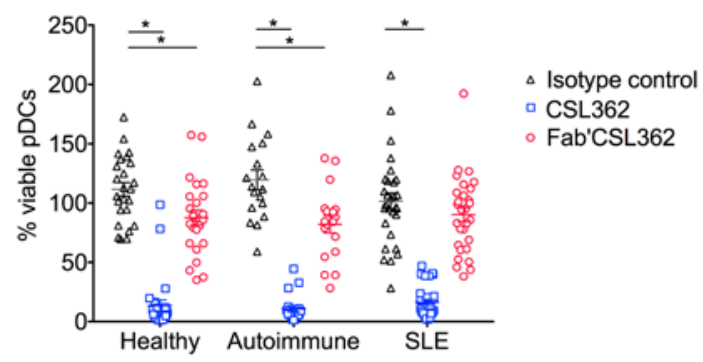

D

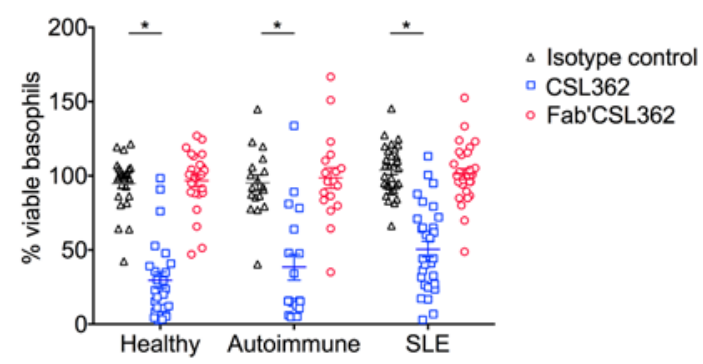

E

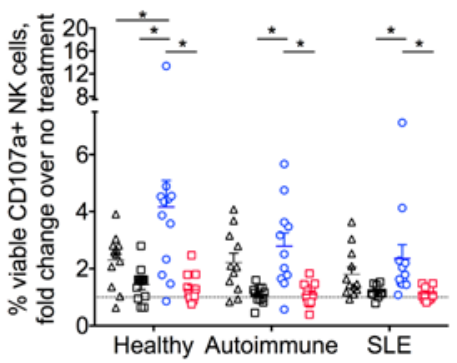

$\triangle$ Isotype control

- Isotype control 2

- CSL362

- Fab'CSL362

Figure 1. CSL362 depletes CD123 ${ }^{\text {hi }}$ plasmacytoid dendritic cells and basophils and activates NK cells. (A) CD123 expression on peripheral blood cells in SLE donors $(n=34)$ and healthy $(n=34)$ and autoimmune $(n=20)$ controls, as determined by flow cytometry using Quantibrite-PE beads. The number of CD123 molecules per cell is shown for each donor. (B) Representative flow cytometric analysis from SLE donors of viable plasmacytoid dendritic cells (pDCs) (Sytox Blue-, Lin1', HLA-DR+, BDCA2 ${ }^{++}$) and basophils (Sytox Blue-, Lin1-, CCR3') after 24-hour culture with media alone (no treatment), CSL362, Fab'CSL362, or isotype control. Percentage of viable (C) pDCs and (D) basophils, as determined by flow cytometry, after 24-hour culture with $0.01 \mu \mathrm{M}$ CSL362, Fab'CSL362, or isotype control compared with media alone in SLE $(n=30)$, healthy $(n=25)$, and autoimmune donors $(n=18)$. (E) Fold change of percentage viable CD107a+ NK cells, as determined by flow cytometry, after 18- to 21-hour culture with $0.01 \mu$ M CSL362, Fab'CSL362, or 2 isotype controls compared with media alone for SLE $(n=12)$, healthy $(n=12)$, and autoimmune $(n=11)$ donors. Isotype control contains the same modified Fc region as CSL362, isotype control 2 contains an unmodified IgG1-Fc. Data are expressed as mean \pm SEM, ${ }^{*} P<0.05$ (Mann Whitney test).

to replace TLR7- or TLR9-induced IFN- $\alpha$ production in the absence of pDCs and, importantly, also show that global IFN- $\alpha$ production is not affected by CSL362, as cells other than pDCs can produce IFN- $\alpha$ in response to TLR3, TLR4, TLR5, and TLR8 stimulation.

We next developed an IFN gene score based on the targeted IFN gene "signature" (described above) to assess drug efficacy. The panel of 11 IFN-inducible genes was incorporated into a single gene score to facilitate comparison between SLE donors and healthy donors. The derived IFN gene score was calculated as the average of the $\log _{2}$ fold change in expression of the 11 genes compared with that of a universal healthy control. The gene score for most healthy donors was close to 0, whereas SLE donors had an average gene score of approximately 3.0, representing an approximately 8-fold change (Figure 3A), consistent with prior exposure to type I IFN in this population.

The effect of CSL362 on TLR7- and TLR9-induced IFN- $\alpha$ production and the IFN gene score was subsequently examined in SLE, autoimmune, and healthy donors. TLR9-induced IFN- $\alpha$ production was essentially negated in all donors with CSL362 pretreatment but not with Fab'CSL362 or isotype control pretreatment (Figure 3B). CSL362 pretreatment also reduced the IFN gene score as compared with isotype control (Figure 3C). Similar effects were observed in TLR7-responsive donors (Supplemental Figure 3).

Immune complexes or other components of SLE serum may contribute to IFN- $\alpha$ production independently of TLR7 and TLR9 activation. Therefore, we evaluated IFN- $\alpha$ production induced by SLE serum 


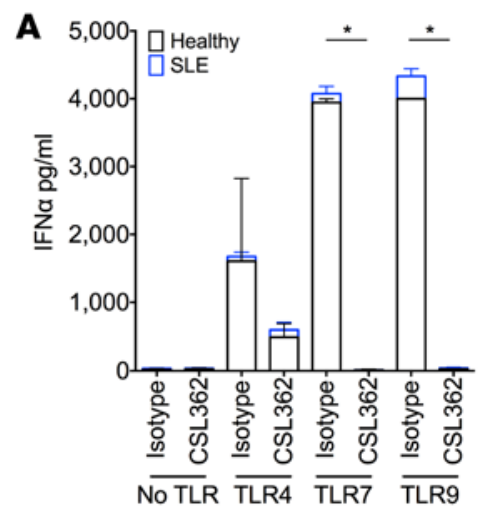

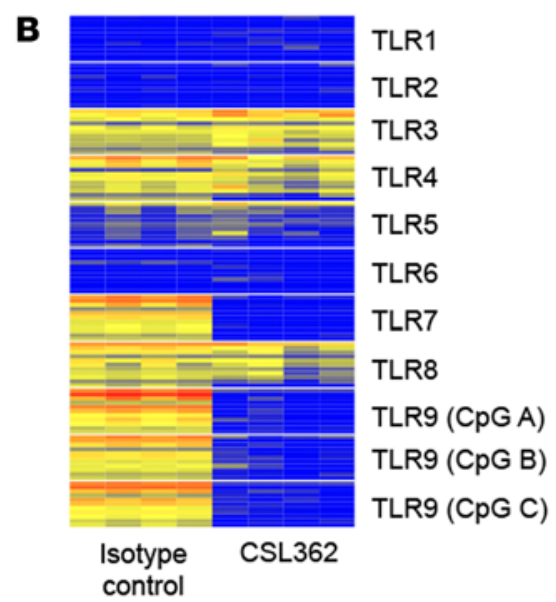
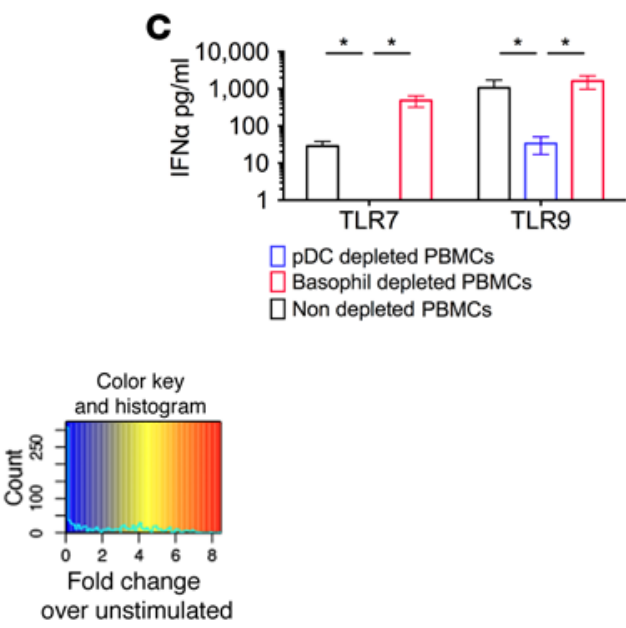

Figure 2. Depletion of plasmacytoid dendritic cells by CSL362 potently and specifically inhibits TLR7- and TLR9-induced IFN- $\alpha$ production and IFN- $\alpha$ inducible gene expression. (A) IFN- $\alpha$ production, as measured by ELISA, in response to TLR4, TLR7, and TLR9 stimulation following CSL362 or isotype control pretreatment of healthy and SLE donor ( $n=3$ each) peripheral blood mononuclear cells (PBMCs). (B) Expression of 11 IFN-inducible genes (IFI44L, IFIT1, IFIT3, IRF7, ISG15, MX1, MX2, OAS1, OAS2, SERPING1, and XAF1), as determined by qPCR, in response to TLR1-9 agonist stimulation following CSL362 or isotype control pretreatment in healthy donor PBMCs $(n=4)$. (C) IFN- $\alpha$ production, as determined by ELISA, from healthy $(n=2)$ and SLE $(n=2)$ donor PBMCs that were depleted either of plasmacytoid dendritic cells (pDCs) or basophils and stimulated with TLR7 (imiquimod) or TLR9 (CpG C) agonist. Data are expressed as mean $\pm \mathrm{SEM},{ }^{*} P<0.05$ (Mann Whitney test).

with varying levels of anti-dsDNA antibody titers. Sera with low (3.4-7.1 IU/ml), medium (91.8-104.3 $\mathrm{IU} / \mathrm{ml}$ ), or high anti-dsDNA $(>470 \mathrm{IU} / \mathrm{ml})$ antibody levels was used to stimulate $\mathrm{IFN}-\alpha$ production in healthy donor PBMCs. Sera from donors with a high anti-dsDNA antibody level intrinsically contained a small amount of detectable IFN- $\alpha$ and when cultured with healthy PBMCs was able to stimulate further IFN- $\alpha$ production (Figure 3D). Importantly, the IFN- $\alpha$ that was produced by serum stimulation was completely inhibited by CSL362 (Figure 3D). Sera from the donors with low and medium anti-dsDNA antibody levels was not able to stimulate detectable IFN- $\alpha$ production when cultured with healthy donor PBMCs; however, it was able to upregulate expression of IFN-inducible genes, and this expression was also decreased by pretreatment with CSL362 (Figure 3E).

Using published DNA sequences and recombinant methods, we produced and purified 2 anti-IFN- $\alpha$ mAbs for comparison to CSL362. Both anti-IFN- $\alpha$ mAbs neutralized TLR7- and TLR9-stimulated IFN- $\alpha$, with $>90 \%$ neutralization at doses $\geq 10 \mu \mathrm{g} / \mathrm{ml}$. However, CSL362 reduced IFN- $\alpha$ more potently, with complete inhibition at equivalent doses (Figure 4A). Although the anti-IFN- $\alpha$ mAbs neutralized IFN- $\alpha$, these had minimal impact on reducing TLR-induced IFN-upregulated gene expression in the IFN gene "signature," as compared with CSL362 (Figure 4B). This may be because activated pDCs produce more than one type of IFN that may contribute to the gene signature. Elevated levels of multiple type I IFN subtypes (IFNA, IFNB1, IFNW, IFNE) as well as type III IFN (IFNL1, IFNL2, IFNL3) levels were seen by RNA sequencing of isolated pDCs stimulated with TLR7 or TLR9 agonists (Figure 4C). Type II IFN (IFNG) levels were not differentially expressed. We then assessed the effect of CSL362 on TLR7- and TLR9induced type III IFN (IFN- $\lambda 1$, IFN- $\lambda 2$ and IFN- $\lambda 3$ ) levels in 9 healthy donors and 6 SLE donors. Three healthy donors and two SLE donors responded to TLR9 (CpG) stimulation with detectable ( $>50 \mathrm{pg} / \mathrm{ml})$ type III IFN levels, as assessed by ELISA, which were reduced to negligible levels in all donors by CSL362 treatment (Figure 4D). In the 3 healthy donors and 3 SLE donors that responded to TLR7 (imiquimod) stimulation, CSL362 treatment led to a decreased production of IFN- $\lambda$ in these donors (Figure 4D) that approached significance $(P=0.09)$. In contrast, there was no significant inhibition by CSL362 of TLR7- or TLR9-stimulated type II (IFN- $\gamma$ ) production in these same donors (Figure 4D).

CSL362 inhibits TLR7- and TLR9-induced plasmablast expansion and proliferation by depletion of pDCs. Virally activated pDCs promote CD40L-stimulated plasmablast differentiation through IFN- $\alpha$ and IL-6 (34). We found that TLR7 and TLR9 stimulation were both able to expand the same plasmablast population $\left(\mathrm{CD} 19^{+}, \mathrm{CD} 27^{++}, \mathrm{CD} 20^{-}, \mathrm{CD} 38^{++}\right)$, reaching maximal expansion at day 6 (Supplemental Figure 4). Pretreatment with CSL362, but not isotype control, inhibited this expansion in both healthy and SLE donors (Figure 5, A-C), although inhibition in SLE donors was less robust than in the healthy controls. 
A

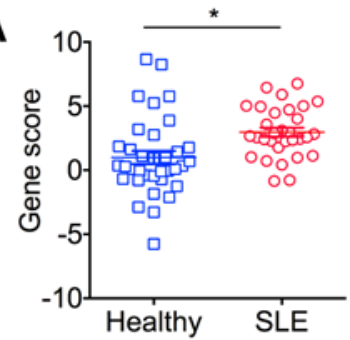

C
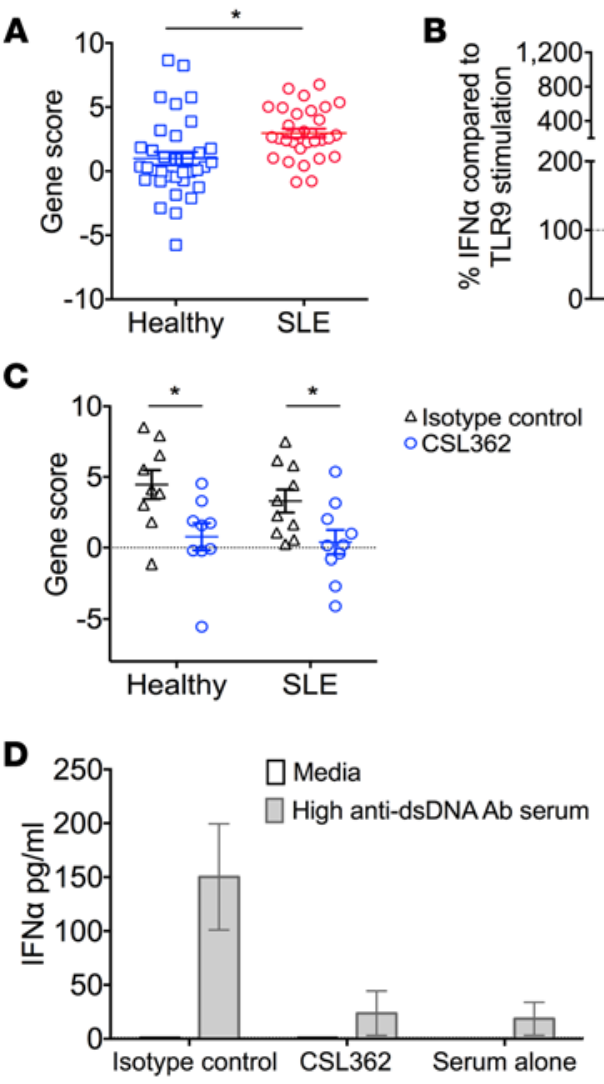

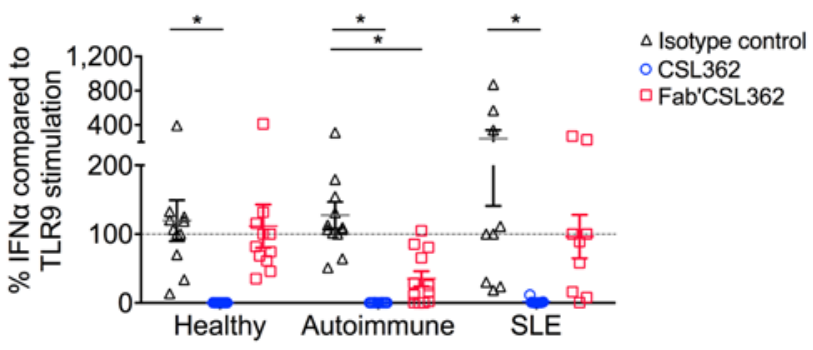

E

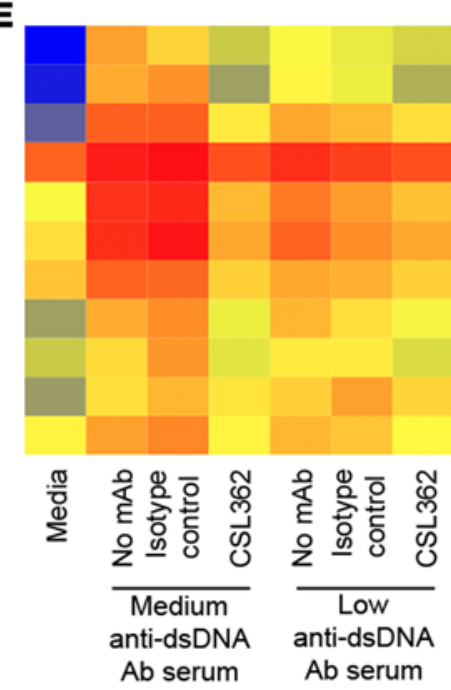

IFI44L

IFIT1

IFIT3

IRF7

ISG15

MX1

MX2

OAS1

OAS2

SERPING1

XAF1

.

Figure 3. CSL362 inhibits TLR7, TLR9, and SLE serum-induced IFN- $\alpha$ production and IFN-inducible gene expression in SLE, autoimmune, and healthy donors. (A) IFN-inducible gene expression, expressed as a single gene score, in SLE $(n=31)$ and healthy $(n=35)$ donor whole blood. Gene score represents the average $\log _{2}$ fold change in expression of 11 IFN-inducible genes (IFI44L, IFIT1, IFIT3, IRF7, ISC15, MX1, MX2, OAS1, OAS2, SERPING1, and XAF1) compared with a universal healthy donor. (B) IFN- $\alpha$ production from SLE $(n=9)$, autoimmune $(n=12)$, and healthy $(n=11)$ donor peripheral blood mononuclear cells (PBMCs) and (C) IFN-inducible gene expression, expressed as a single gene score for SLE $(n=10)$ and healthy $(n=9)$ donor PBMCs stimulated with TLR9 agonist (CpC C), following CSL362, Fab'CSL362 (B, only) or isotype control pretreatment. Gene score represents the average log fold change in expression of 11 IFN-inducible genes (IFI44L, IFIT1, IFIT3, IRF7, ISG15, MX1, MX2, OAS1, OAS2, SERPING1, and XAF1) for each treatment compared with no treatment. (D) IFN production $(n=3)$ from healthy donor PBMCs pretreated with CSL362 or isotype control and then stimulated with $50 \%$ SLE serum containing high anti-dsDNA antibody levels. The level of IFN- $\alpha$ detectable in the serum alone (no PBMC control) is shown in the "serum alone" condition. (E) IFN-inducible gene expression in healthy donor PBMCs stimulated with 50\% SLE serum containing low or medium anti-dsDNA antibody levels, following CSL362 or isotype control pretreatment. IFN-inducible gene expression determined by qPCR, IFN- $\alpha$ production determined by ELISA. Data are expressed as mean $\pm \mathrm{SEM},{ }^{*} P<0.05$ (Mann Whitney test).

TLR9 stimulation induced a greater plasmablast expansion than TLR7 and a higher concentration of CSL362 was required to suppress this TLR9-stimulated response (Figure 5D). Using CFSE staining, we showed that cellular proliferation in response to TLR7 and TLR9 agonists was inhibited by CSL362 in both memory B cells and plasmablasts (Figure 5E). This suggests that either pDCs or basophils provide a proliferative stimulus to B cells when activated by TLR7 or TLR9 agonists. Interestingly, the induction of naive B cell proliferation by TLR9 stimulation was not inhibited by CSL362, suggesting that TLR9-induced proliferation of naive $\mathrm{B}$ cells is a direct effect.

To determine the contribution of pDCs and basophils to plasmablast expansion, we reconstituted each cell type separately into CSL362-pretreated cultures. Reconstitution with pDCs, but not basophils, was able to restore TLR9-induced plasmablast expansion (Figure 6, A and B). To determine whether the reconstitution effect was direct or via secreted factors, conditioned media (CM) from pDCs was added to CSL362-pretreated cultures. CM from pDCs stimulated with TLR9 agonist (CpG) was able to restore plasmablast expansion (Figure 6C) and did so in a dose-dependent manner (Figure 6D), whereas CM from nonstimulated pDCs did not induce plasmablast expansion. These data suggested that a soluble factor or factors produced by $\mathrm{CpG}$-activated pDCs promote plasmablast expansion. Similar experiments were performed using TLR7 agonist imiquimod as a stimulus; however, in these experiments, there was insufficient plasmablast expansion to allow a comparison of nonreconstituted and reconstituted cultures. 
A

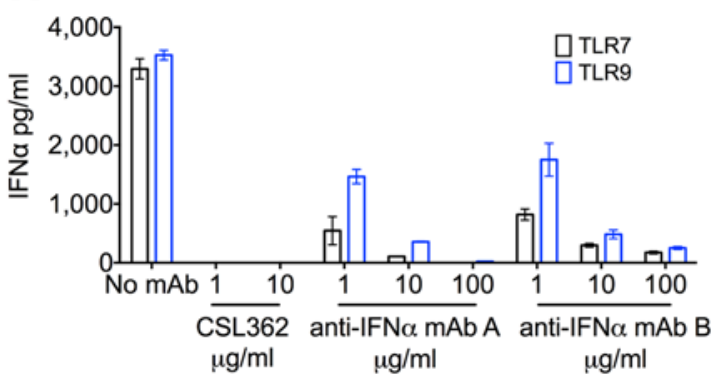

B

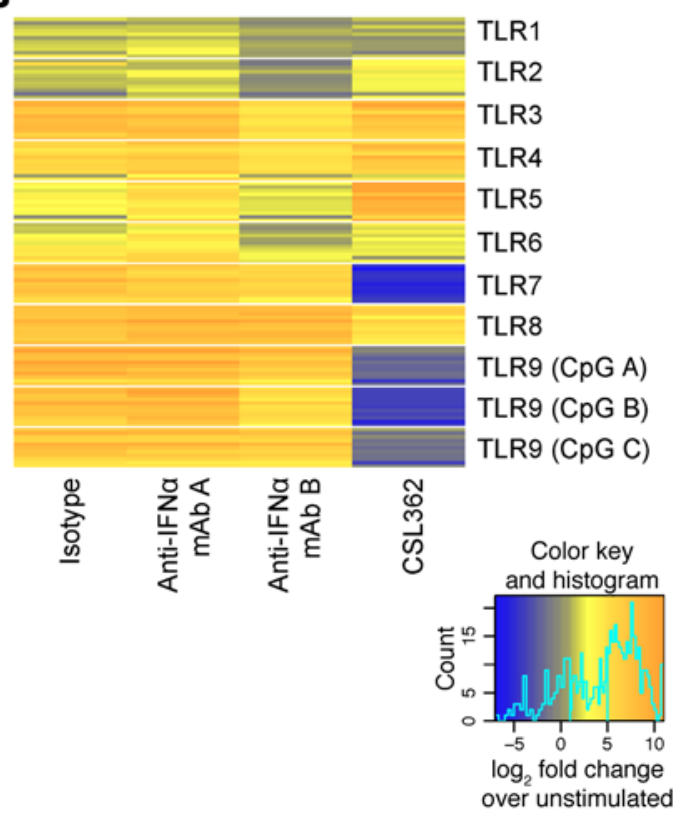

C
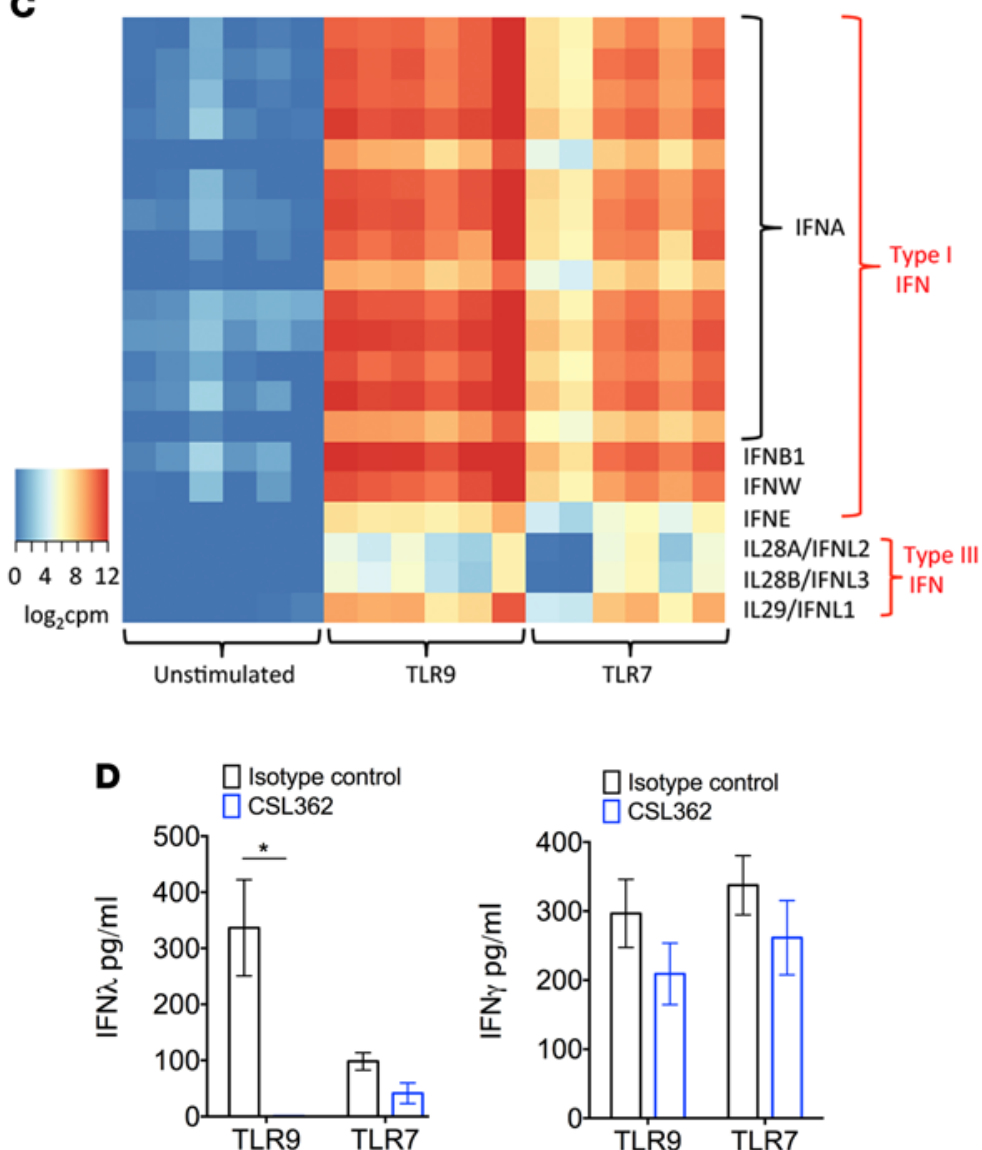

Figure 4. CSL362 more effectively inhibits TLR7- and TLR9-stimulated IFN production and IFN-upregulated gene expression compared with type I IFN blockade. (A) IFN- $\alpha$ production in response to TLR9 (CPC C) or TLR7 (imiquimod) stimulation of healthy donor peripheral blood mononuclear cells (PBMCs) $(n=2)$ and (B) expression of 11 IFN-inducible genes (IFI44L, IFIT1, IFIT3, IRF7, ISC15, MX1, MX2, OAS1, OAS2, SERPING1, and XAF1) in response to TLR1-9 stimulation in healthy donors $(n=4)$, following pretreatment with CSL362, isotype control, or 2 anti-IFN- $\alpha$ mAbs. IFN-inducible gene expression determined by qPCR, IFN- $\alpha$ production determined by ELISA. Data are expressed as mean \pm SEM, $P<0.05$ (Mann Whitney test). (C) Expression of type I (IFNA, IFNB1, IFNW, IFNE) and type III (IFNL1, IFNL2, IFNL3) IFN subtypes in response to TLR9 or TLR7 stimulation in healthy donors ( $n=6$ ), as assessed by RNA sequencing of isolated pDCs. Type II IFN (IFNG) was not differentially expressed. (D) TLR7- and TLR9-stimulated IFN- $\lambda$ (TLR9 $-n=3$ healthy, $n=2$ SLE; TLR7 $-n=3$ healthy and $n=3$ SLE) and IFN $-\gamma(n=9$ healthy, $n=6$ SLE) production, following pretreatment with CSL362 or isotype control. IFN- $\lambda$ and IFN- $\gamma$ production determined by ELISA. Data are expressed as mean $\pm \mathrm{SEM},{ }^{*} P<0.05$ (Mann Whitney test).

To identify the soluble factor or factors elaborated by stimulated $\mathrm{pDCs}$ that promote plasmablast expansion, Luminex and ELISA analysis of the CM was undertaken. Twenty-eight cytokines were analyzed, and of these, only IFN- $\alpha$, IL-6, and TNF- $\alpha$ were differentially elevated in TLR9-stimulated pDC CM (Supplemental Figure 5) when compared with CM from TLR9-stimulated basophils or unstimulated pDC CM. Furthermore, inhibition of IFN- $\alpha$ and IL- 6 , but not TNF- $\alpha$, by neutralizing mAbs prevented restoration of plasmablast expansion with TLR9-stimulated pDC CM (Figure 6E). These data indicate that IFN- $\alpha$ and IL-6 produced by CpG-activated pDCs stimulate plasmablast expansion.

We compared CSL362 to 2 anti-IFN- $\alpha$ mAbs for the ability to inhibit plasmablast expansion stimulated by either TLR7 or TLR9 agonists or CD40 ligand (CD40L), which induces plasmablast expansion through a non-TLR-mediated mechanism and does not activate $\mathrm{pDCs}$ or induce IFN- $\alpha$ production. CSL362 inhibited TLR-induced, but not CD40L-induced, plasmablast expansion (Figure 6F), again suggesting that CSL362 does not directly affect plasmablast expansion. Inhibition by CSL362 was associated with depletion of pDCs and basophils. In contrast, the anti-IFN- $\alpha$ mAbs did not significantly reduce plasmablast expansion or deplete $\mathrm{pDCs}$ (Figure 6F). It is possible that the concentration of anti-IFN- $\alpha$ mAbs used was not sufficient to neutralize all pDC-produced IFN- $\alpha$. This suggests that depleting pDCs more efficiently pre- 
A

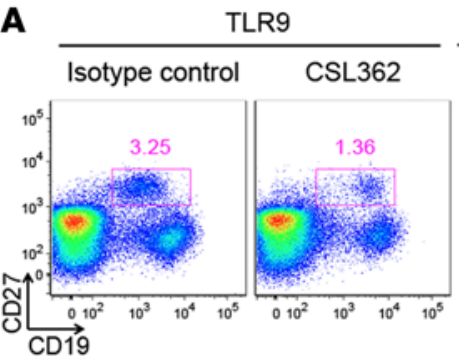

TLR7

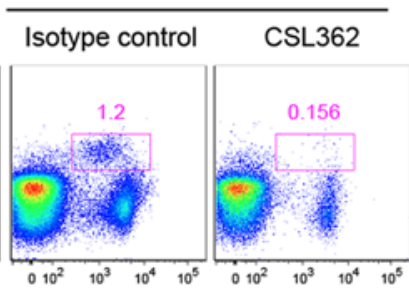

B
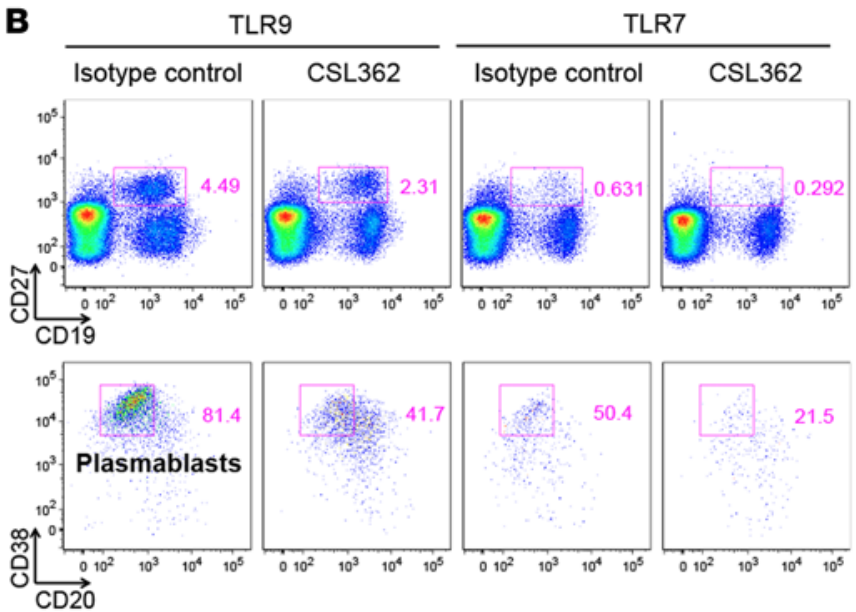
$\overrightarrow{C D 20}$
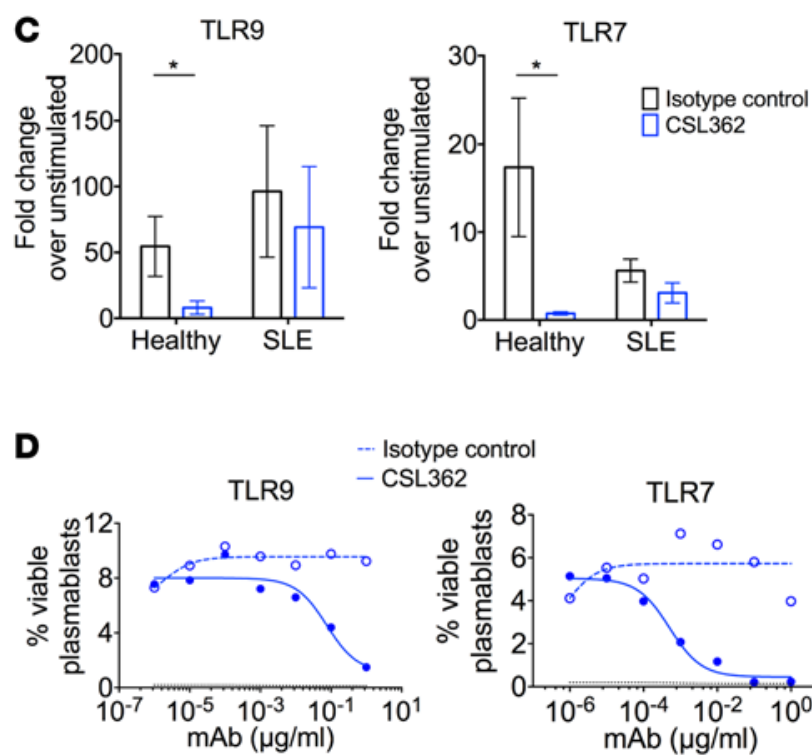

$$
\begin{aligned}
& \text {-. Isotype control } \\
& \text { - CSL362 }
\end{aligned}
$$

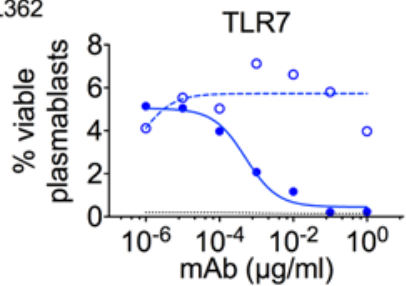

\section{E}
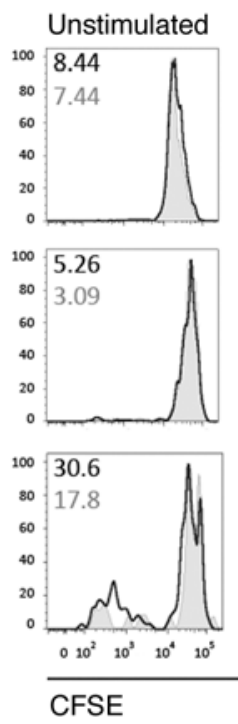
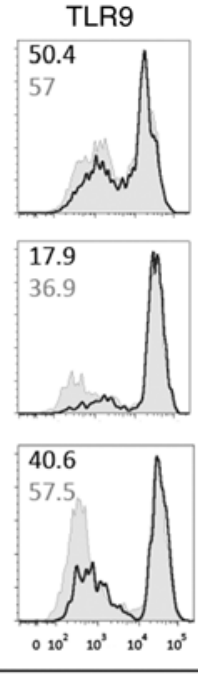
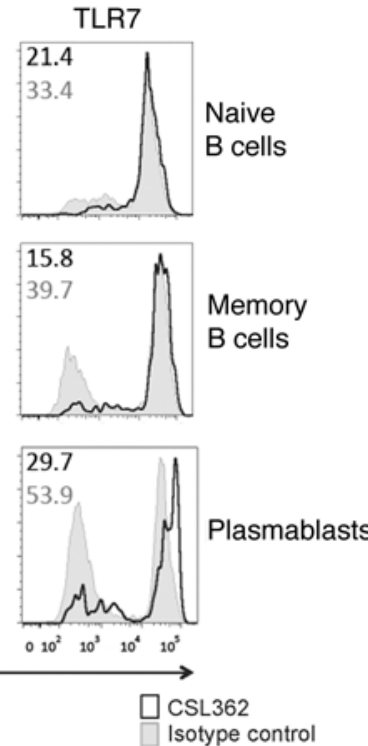

Figure 5. CSL362 inhibits TLR7- and TLR9-stimulated plasmablast expansion and proliferation. Representative flow cytometric analysis from (A) healthy and (B) SLE donors of viable plasmablasts (Sytox Blue ${ }^{-}$, CD19+, CD27 $7^{++}$, CD20-, CD38 ${ }^{++}$) after stimulation with TLR9 (CpG C) or TLR7 (imiquimod) agonists, following CSL362 or isotype control pretreatment. (C) Viable plasmablasts, as expressed as fold change compared with no treatment, following CSL362 or isotype control pretreatment in the presence of TLR7 or TLR9 stimulation for SLE $(n=9)$ and healthy $(n=10)$ donors. (D) Representative data from a healthy donor showing the percentage of viable plasmablasts, as determined by flow cytometry, after stimulation with CpC or imiquimod, following pretreatment with CSL362 or isotype control at varying doses $(n=2)$. (E) Representative flow cytometric analysis of the proliferation of naive and memory B cells and plasmablasts, as assessed by CFSE labeling, in the presence CpC or imiquimod, following CSL362 or isotype control pretreatment ( $n=2)$. Percentages of proliferating (CFSE-negative) cells are shown for each condition. Data in $\mathbf{C}$ and $\mathbf{D}$ are expressed as mean \pm SEM, ${ }^{*} P<0.05$ (Mann Whitney test).

vents TLR-induced plasmablast expansion than inhibiting IFN- $\alpha$ alone, possibly because activated pDCs produce additional cytokines, such as IL-6, that promote plasmablast expansion, as demonstrated above.

s.c. administration of CSL362 to cynomolgus macaques depletes pDCs and basophils in vivo and inhibits TLR9. induced IFN- $\alpha$-inducible gene expression. We next sought to determine the in vivo effect of CSL362 on pDCs and subsequent IFN- $\alpha$ production when administered s.c., which is often the preferred route of delivery of biological therapeutics in chronic diseases such as SLE. CSL362 does not cross react with mouse CD123; however, there is a high degree of sequence homology between human and cynomolgus macaque CD123, which is also highly expressed on cynomolgus macaque pDCs and basophils. In addition, CSL362 demonstrated similar affinity between human and cynomolgus macaque CD123 and Fc $\gamma$ Rs and comparable activity in cell-based assays (30). We treated naive cynomolgus monkeys with a single s.c. injection of CSL362 at varying doses $(1,10,30 \mathrm{mg} / \mathrm{kg})$. Maximal serum concentrations of CSL362 were detected at 48 hours $(\sim 12,190$, and $380 \mu \mathrm{g} / \mathrm{ml}$ at doses of 1,10 , and $30 \mathrm{mg} / \mathrm{kg}$, respectively). Serum CSL362 was maintained 
A $\operatorname{CSL} 36$

pDCs

Basophils
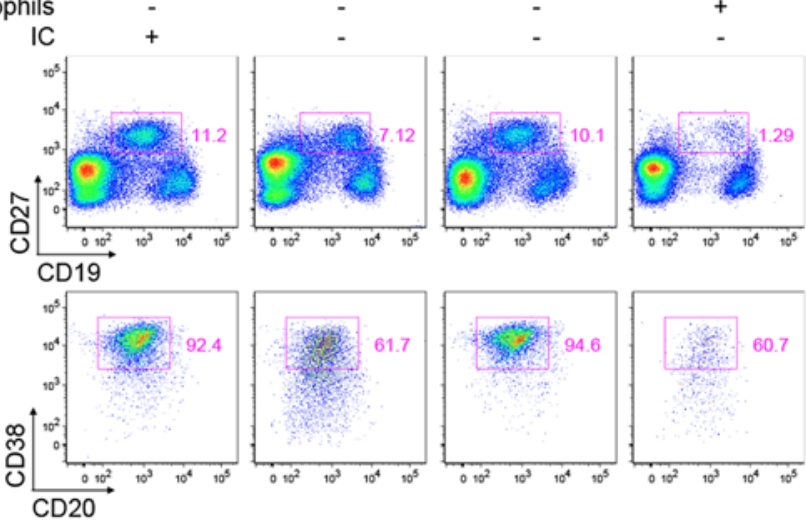

c

CSL362
Neg CM
CpG CM
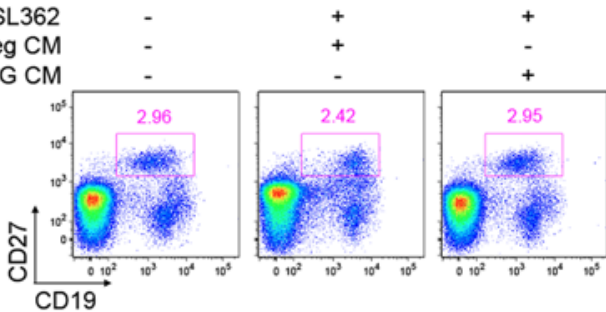

D

- CSL362 + neg CM

$\rightarrow \mathrm{CSL} 362+\mathrm{CpG}$ CM

+ Isotype control $+\mathrm{CpG} \mathrm{CM}$

$\oplus \mathrm{CpG} \mathrm{CM}$
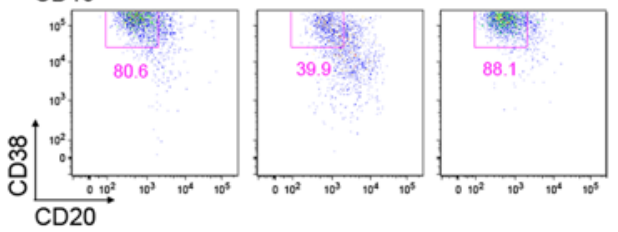

$\mathbf{F}$
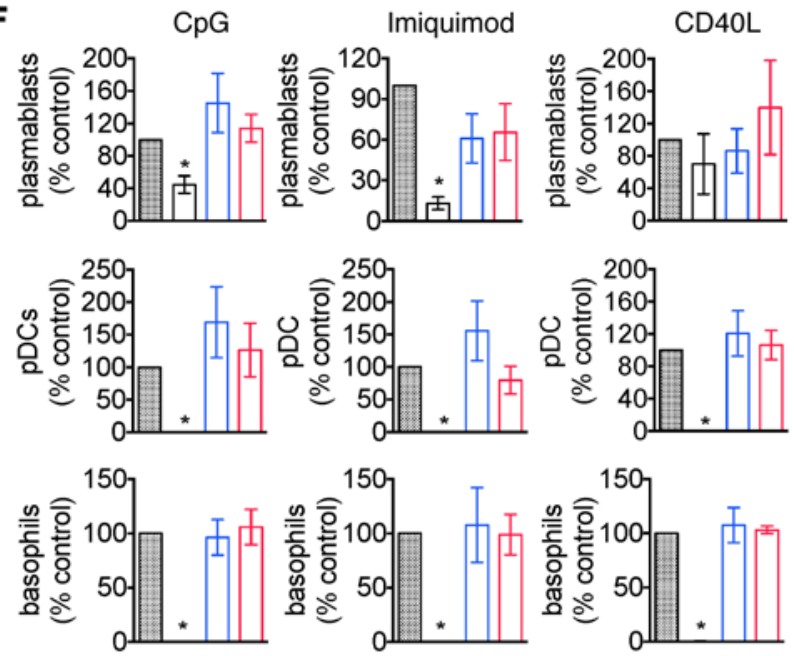

Isotype control

$\square \operatorname{CSL} 362$

Anti-IFN $\alpha$ mAb A

$\square$ Anti-IFN $\alpha$ mAb B
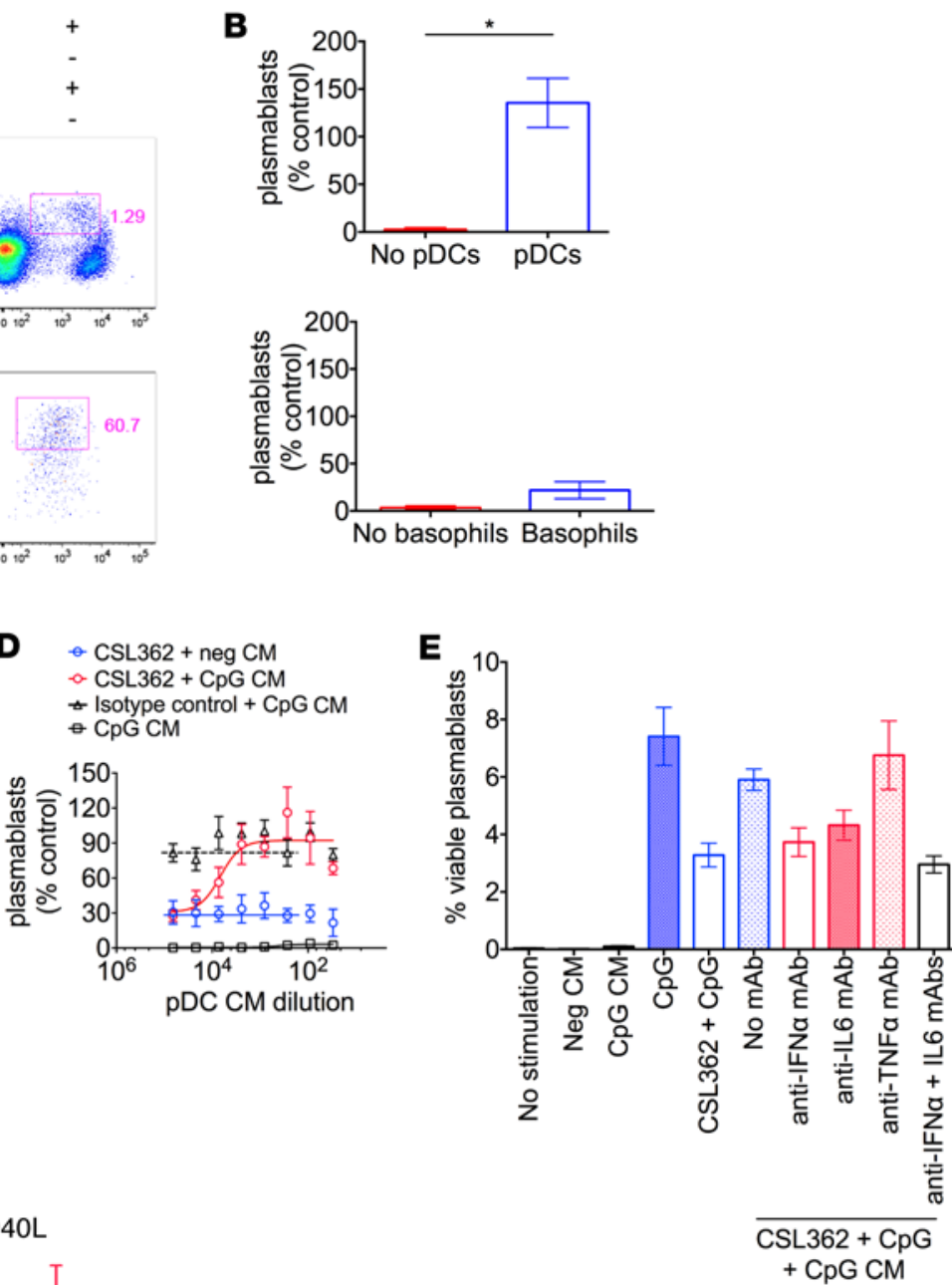

Figure 6. Reconstitution of CSL362-treated peripheral blood mononuclear cells with plasmacytoid dendritic cells or plasmacytoid dendritic cell-conditioned media, but not basophils, restores TLR9-induced plasmablast expansion. (A) Representative flow cytometric analysis from a healthy donor of viable plasmablasts (Sytox Blue-, $\mathrm{CD}_{19}+, \mathrm{CD}^{27^{++}}, \mathrm{CD}^{-} \mathrm{O}^{-}, \mathrm{CD}^{+++}$) following reconstitution of plasmacytoid dendritic cells (pDCs) or basophils into CSL362 or isotype control (IC) pretreated and TLR9-stimulated (CpGstimulated) peripheral blood mononuclear cell (PBMC) cultures. (B) Viable plasmablasts, expressed as a percentage compared with isotype control, as determined by flow cytometry, following reconstitution of pDCs or basophils into CSL362-pretreated and TLR9-stimulated PBMC cultures ( $n=4$ healthy donors). (C) Representative flow cytometric analysis from a healthy donor of viable plasmablasts in PBMCs stimulated with TLR9 agonist (CpC), following CSL362 or isotype control pretreatment. Cultures were supplemented with conditioned media from isolated pDCs cultured with TLR9 agonist (CpC CM) or media alone (neg CM). (D) Viable plasmablasts, determined by flow cytometry, after reconstitution of CSL362 or isotype control-pretreated healthy PBMCs $(n=4)$ with increasing concentrations of pDC-conditioned media (CpC CM or neg CM) and stimulation with TLR9 agonist. Data are expressed as a percentage compared with media alone. (E) Viable plasmablasts, determined by flow cytometry, in TLR9-stimulated PBMCs (CpC), following CSL362 pretreatment. Cultures were supplemented with $\mathrm{CpC} C \mathrm{CM}$ in the absence of neutralizing antibodies (no mAb) or presence of anti-IFN, anti-IL-6, or anti-TNF antibodies ( $n=3$ healthy donors). (F) Viable plasmablasts, pDCs, and basophils, determined by flow cytometry, from healthy donors $(n=4)$, expressed as a percentage compared with media alone, following pretreatment with CSL362, isotype control, or 2 anti-IFN- $\alpha$ mAbs ( $A$ and $B$ ) and stimulation with TLR9 agonist (CpG), TLR7 agonist (imiquimod), or CD4OL. Data are expressed as mean $\pm \mathrm{SEM},{ }^{*} P<0.05$ (Mann-Whitney test). 
A

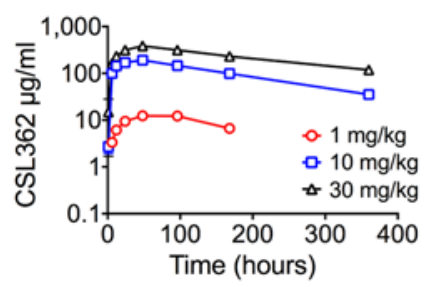

C

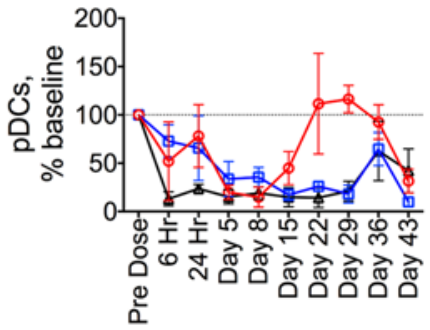

D

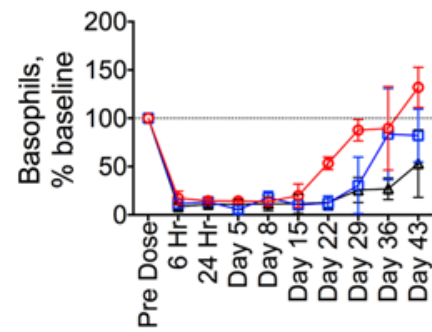

E

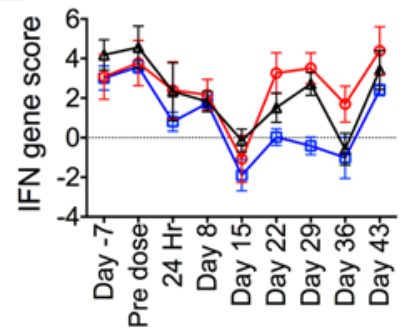

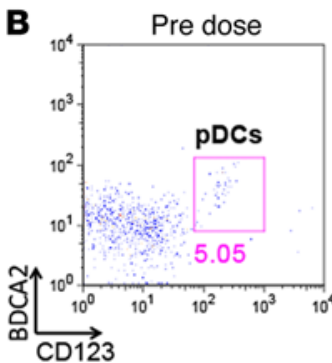

$6 \mathrm{Hr}$
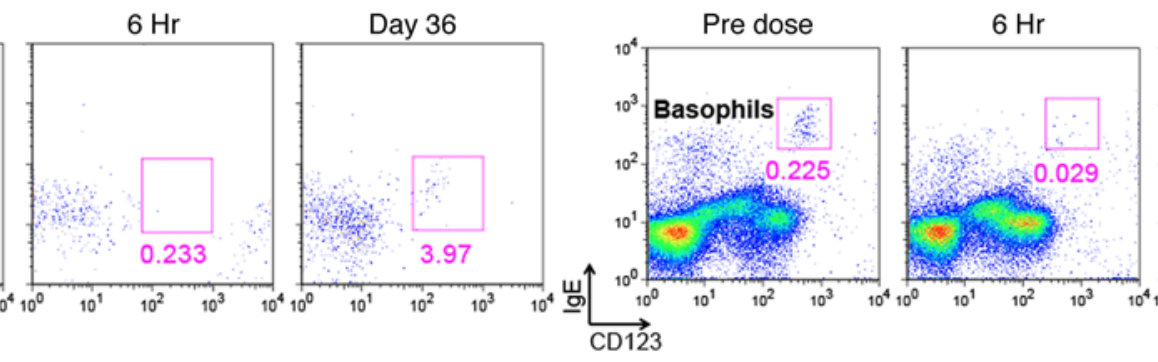

Day 36

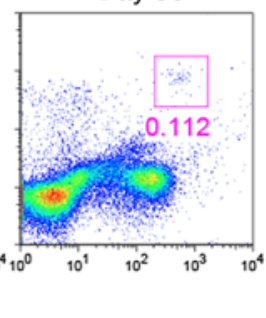

Figure 7. s.c. administration of CSL362 to cynomolgus macaques depletes plasmacytoid dendritic cells and basophils and inhibits TLR9-induced IFN-inducible gene expression. (A) Serum concentration of CSL362, determined by ELISA at various time points following s.c. administration of CSL362 (at doses of 1, 10, and $30 \mathrm{mg} / \mathrm{kg}$ ) to cynomolgus macaques. (B) Representative flow cytometric analysis showing plasmacytoid dendritic cells (pDCs) (Lin1-,

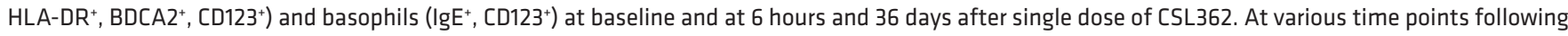
s.c. administration of CSL362, (C) pDCs and (D) basophils, in peripheral blood, expressed as a percentage of baseline (pretreatment), as determined by flow cytometry. (E) Expression of 6 IFN-inducible genes (IFI35, IFIT1, IRF7, MX1, MX2, OAS1) as a single gene score in peripheral blood mononuclear cells cultured with TLR9 agonist (CpG), as determined by qPCR. The gene score represents the average $\log _{2}$ fold change in the 6 IFN-inducible genes over unstimulated control. Data are expressed as mean \pm SEM for $n=3$ animals per dosing group.

above $1 \mu \mathrm{g} / \mathrm{ml}$ at day 7 for the $1 \mathrm{mg} / \mathrm{kg}$ dose and above $30 \mu \mathrm{g} / \mathrm{ml}$ for 14 days at the higher doses (Figure 7A). pDC depletion was achieved as early as 6 hours after administration for the highest dose and was maximal between days 5 and 15 for all doses (Figure 7, B and C). Similarly, basophils were also depleted by s.c. administration of CSL362 (Figure 7, B and D). These effects followed the peak serum drug levels and were maintained for 4 weeks at the 2 highest doses for pDCs and for 2 weeks for basophils (Figure 7, B-D). We assessed the effect of in vivo pDC depletion by CSL362 on IFN-induced gene expression by stimulating PBMCs, isolated at various time points after CSL362 administration, with CpG for 24 hours ex vivo. A decrease in IFN-inducible gene expression was observed following CSL362 administration (Figure 7E). The decrease in serum drug levels from day 22 onwards correlated with recovery of pDC numbers and an associated increase in $\mathrm{CpG}$-stimulated IFN- $\alpha$-inducible gene expression. Importantly, s.c. administration of CSL362 was well tolerated at all doses, and no overt toxicity was observed.

\section{Discussion}

We have shown that a humanized mAb targeting IL-3R $\alpha$ (CSL362) has a unique activity profile, with effects against several key cell types and cytokines involved in SLE, thereby providing a rationale for CSL362's evaluation as a therapeutic agent in this disease. CSL362 potently depletes pDCs, leading to selective abrogation of TLR7, TLR9, and SLE serum-induced IFN- $\alpha$ production and reduced expression of a panel of IFN- $\alpha$-inducible genes. Of note, others have shown that depletion of pDCs ameliorates disease in murine models of lupus $(17,18)$, suggesting that pDC depletion in human SLE may be an effective therapeutic strategy. In addition, we show that CSL362 also depletes basophils, inhibits TLR7- and TLR9stimulated plasmablast expansion, neutralizes IL-3-mediated pDC survival, and has biological activity in vivo in nonhuman primates after s.c. administration. $\mathrm{pDC}$ and basophil depletion by CSL362 is dependent upon ADCC and NK cell activation. NK cells in SLE have an activated phenotype but normal ADCC capability, despite reduced CD16 expression (35). Importantly, we show that NK cells derived from patients with SLE can still be activated through the modified IgG1-Fc portion of CSL362, which is critical to cell depletion through enhanced ADCC.

Our data suggest that depletion of pDCs, rather than blockade of IFN- $\alpha$ alone, may be a more effective 
strategy to inhibit IFN-driven processes, such as gene expression and plasmablast expansion, in response to stimuli, such as immune complexes, that activate TLR7 and TLR9 in SLE. We have shown that by depleting pDCs, production of IFN types other than type I may be inhibited. Of particular interest is type III IFN, for which there is some evidence of aberrant regulation in SLE, with elevated serum levels compared with healthy controls (36). Increased levels of type III IFN were correlated with disease activity, anti-dsDNA antibody levels, glomerulonephritis, and arthritis (37). Although type III IFNs signal through a separate receptor complex than that of type I IFNs, they share common signaling pathways and, as a result, have been postulated to be responsible for ongoing disease activity, despite type I IFN blockade (38). Inhibition of multiple IFN types may therefore confer therapeutic advantage in these situations.

SLE is characterized by autoreactive antibodies, which reflect aberrant activation of $\mathrm{B}$ cells and differentiation into antibody-producing plasma cells. Our data suggest that $\mathrm{pDC}$ depletion as a therapeutic strategy may potentially combat two major pathogenic targets in SLE simultaneously, namely inhibiting IFN- $\alpha$ production and autoantibody production. We have shown that, by depleting pDCs with CSL362, plasmablast expansion and proliferation in response to TLR7 and TLR9 agonists is inhibited, although inhibition in SLE donors appears to be less robust than in healthy donors. Plasmablast expansion occurred in response to soluble factors released by activated pDCs, specifically, IFN- $\alpha$ and IL-6, confirming the importance of IFN- $\alpha$ and IL- 6 in promoting plasmablast expansion found previously in a virally activated, CD40L-dependent system (34). Specific depletion of pDCs in murine models of SLE has not been found to alter peripheral plasma cell numbers; however, germinal centers in spleens were abolished, and there was a reduction in anti-dsDNA and anti-RNA antibodies $(17,18)$. It is possible that depleting pDCs may differentially effect short-lived compared with long-lived plasma cells in different compartments. Interestingly, we found that TLR9 agonist CpG C was a more robust ex vivo stimulus of plasmablast expansion than TLR7 agonist imiquimod and that the concentration of CSL362 required to inhibit imiquimod-induced plasmablast expansion closely followed that required to deplete $\mathrm{pDCs}$ and basophils. This suggests that the contribution of $\mathrm{pDCs}$ and soluble factors produced by $\mathrm{pDCs}$ to TLR7-stimulated plasmablast expansion may be greater than for TLR9.

The ability of CSL362 to deplete basophils and inhibit IL-3 signaling may be of additional benefit. Though basophils in SLE have been less studied compared with pDCs, they can be activated by IgE-containing immune complexes. Upon activation, basophils may augment autoantibody production by eliciting a Th2 response and production of the B cell survival factor BAFF $(31,39)$. Basophil depletion alleviated nephritis in a murine lupus model (31), and therapeutic targeting of basophils in SLE is currently being explored in a phase I trial of an anti-IgE mAb, omalizumab (clinical trial NCT01716312). IL-3 is a known maturation and survival factor for pDCs (32) and basophils (40). We found IL-3 blockade with higher doses of Fab'CSL362 depleted pDCs. Although IL-3 has not been extensively studied in SLE, elevated serum IL-3 levels have been reported in active SLE patients (41). More recently, administration of IL-3 in the MRL/lpr murine lupus model was found to exacerbate nephritis, and this was improved by IL-3 blockade, suggesting an important role for IL-3 in the progression of lupus nephritis (42). Therefore, the potential beneficial effects of CSL362 in SLE may extend to its ability to neutralize IL-3 in addition to depletion of pDCs and basophils.

A phase I trial in AML (clinical trial NCT01632852) using intravenously administered CSL362 in doses ranging from 0.3 to $12.0 \mathrm{mg} / \mathrm{kg}$ has recently been completed (43). In that study, there were no increased infections despite rapid ( $\leq 6$ hours after dose) and complete $\mathrm{pDC}$ and basophil depletion at all doses for a fortnightly dosing frequency, which was sustained for $\geq 15$ days for doses $\geq 3 \mathrm{mg} / \mathrm{kg}$; however, a phase II trial will provide data regarding longer-term infection risk. In the current study, we report the use of s.c. administration in cynomolgus monkeys. This approach was taken because s.c. administration has a range of benefits in chronic diseases, such as SLE. Single s.c. administered doses of CSL362 given to cynomolgus macaques were well tolerated, and the biological effects were reversible, with recovery of depleted pDCs and basophils and of IFN-inducible gene expression occurring in step with waning serum drug levels.

In addition to SLE, we have shown that CSL362 was effective ex vivo in depleting pDCs and reducing IFN- $\alpha$ production in patients with a variety of type I IFN-associated autoimmune diseases, including psoriasis, scleroderma, primary Sjogren's syndrome, inflammatory myopathy (44), and rheumatoid arthritis (45). In a recent phase I trial in scleroderma, IFN receptor blockade showed promising results, with near complete inhibition of IFN-stimulated gene expression in peripheral blood and skin (46). In contrast, IFN-0 blockade had no clinical activity against plaque psoriasis (47), although IFN receptor (through which both 
IFN- $\alpha$ and IFN- $\beta$ signal) blockade may be more effective than IFN- $\alpha$ blockade alone. Our data suggest that CSL362 may be a new tool to dissect the role of pDCs, basophils, and IL-3 in various human diseases and highlight its potential role for use in the treatment of SLE and other IFN-dependent disorders.

\section{Methods}

Human subjects. SLE and autoimmune disease control (rheumatoid arthritis and psoriatic arthritis $[n=4$ each], scleroderma $[n=3]$, psoriasis and ankylosing spondylitis $[n=2$ each], seronegative inflammatory arthritis, primary Sjogren's syndrome, polymyositis, granulomatosis with polyangiitis, and minimal change renal disease $[n=1$ each]) blood samples were obtained from adult patients at The Royal Melbourne Hospital. SLE patients fulfilled SLICC classification criteria for SLE (48). Healthy controls were obtained from the Volunteer Blood Donor Registry at The Walter and Eliza Hall Institute of Medical Research. The SELENA-SLEDAI (Safety of Estrogens in Lupus Erythematosus National Assessment Systemic Lupus Erythematosus Disease Activity Index) score (49) was used to evaluate lupus disease activity.

Human sample collection. Whole blood was collected into lithium heparin tubes (BD, catalog 367526) for whole blood and PBMC assays. Serum was collected into SST II Advance tubes (BD, catalog 367958) for storage at $-80^{\circ} \mathrm{C}$ after centrifugation. PAXgene Blood RNA tubes (BD, catalog 762165) were frozen at $-20^{\circ} \mathrm{C}$ for later RNA extraction.

Tissue culture. PBMCs were isolated from whole blood by Ficoll density centrifugation (GE Healthcare Life Sciences, catalog 17-1440-02). Culture of PBMCs was undertaken in RPMI 1640 media (SigmaAldrich, catalog R0083) supplemented with 10\% heat-inactivated fetal calf serum (HyClone GE Healthcare, catalog SH30084.03HI), 2 mM GlutaMAX (Gibco Life Technologies, catalog 35050-0610), and $0.5 \%$ penicillin/streptomycin (Gibco Life Technologies, catalog $15140-122$ ), at $37^{\circ} \mathrm{C}$ with $5 \% \mathrm{CO}_{2}$, unless otherwise stated.

Antibodies, reagents, and flow cytometry. Most flow cytometry antibodies and reagents used were commercially available and are listed in Supplemental Table 2. Only one antibody was not commercially available and was generated in-house. This was an anti-CD123-PE antibody (HU01C2) that was used in the $\mathrm{pDC}$ and basophil staining panels for the cynomolgus macaque study. Flow cytometry data were acquired with a MACSQuant Analyzer (Miltenyi Biotec) or a LSR Fortessa (BD Biosciences) and analyzed with Flowjo software (Treestar). Cell sorting was performed with a FACSAria (BD Biosciences) or FACS Fusion (BD Biosciences).

Cell surface CD123 expression. Whole blood (50-200 $\mu$ l) was stained with antibody cocktails, each of which included anti-CD123 PE. After red blood cell lysis with BD Lysing Solution (BD, catalog 349202), Quantibrite PE beads (BD, catalog 340495) were used to estimate the number of CD123 molecules on the surface of each cell type using the MACSQuant Analyzer. The cell types were defined by the following surface markers: pDCs (Lin1 ${ }^{-}, \mathrm{HLA}_{-} \mathrm{DR}^{+}, \mathrm{BDCA}^{++}$), basophils (Lin1 ${ }^{-}, \mathrm{CCR}^{+}$), mDCs (Lin1 ${ }^{-}$, HLA-

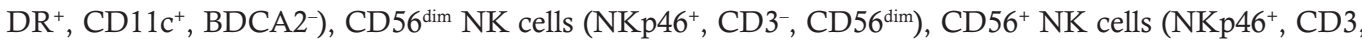
$\mathrm{CD}^{+} 6^{+}$, naive $\mathrm{B}$ cells $\left(\mathrm{CD} 19^{+}, \mathrm{CD} 27^{-}\right)$, memory B cells $\left(\mathrm{CD} 19^{+}, \mathrm{CD} 27^{+}\right)$, plasmablasts $\left(\mathrm{CD} 19^{+}, \mathrm{CD}^{27^{++}}\right.$, $\left.\mathrm{CD} 20^{-}, \mathrm{CD}_{38^{++}}\right)$, classical monocytes $\left(\mathrm{CD}^{-}, \mathrm{CD}^{4} 4^{++}, \mathrm{CD}^{-}\right)$, intermediate monocytes $\left(\mathrm{CD} 3^{-}, \mathrm{CD}^{+4^{++}}\right.$, $\left.\mathrm{CD} 16^{+}\right)$, nonclassical monocytes $\left(\mathrm{CD}^{-}, \mathrm{CD} 14^{+}, \mathrm{CD} 16^{++}\right)$, neutrophils $\left(\mathrm{CD} 16^{+}, \mathrm{CD} 49 \mathrm{~d}^{-}\right)$, eosinophils $\left(\mathrm{CD}^{-}, \mathrm{CD}^{-} \mathrm{d}^{+}\right), \mathrm{CD}^{+} \mathrm{T}$ cells $\left(\mathrm{CD}^{+}, \mathrm{CD}^{+}, \mathrm{CD}^{-}\right)$, and $\mathrm{CD}^{+} \mathrm{T}$ cells $\left(\mathrm{CD}^{+}, \mathrm{CD}^{+}, \mathrm{CD}^{-}\right)$.

Effect of CSL362 on pDCs, basophils, $m D C s$, and $N K, B$, and T cell subsets ex vivo. PBMCs $\left(1.0 \times 10^{6}\right)$ were cultured with CSL362 or isotype control for 24 hours, with doses ranging from 0.001 to $0.1 \mu \mathrm{M}$. The percentage of viable (Sytox Blue ${ }^{-}$) pDCs (Lin1 ${ }^{-}, \mathrm{HLA}_{-} \mathrm{DR}^{+}, \mathrm{BDCA} 2^{++}$), basophils $\left(\mathrm{Lin1}^{-}, \mathrm{CCR}^{+}\right)$, mDCs $\left(\mathrm{Lin}^{-}, \mathrm{HLA}-\mathrm{DR}^{+}, \mathrm{CD} 11 \mathrm{c}^{+}, \mathrm{BDCA2}^{-}, \mathrm{BDCA}^{+}\right), \mathrm{CD}^{\mathrm{dim}} \mathrm{NK}$ cells $\left(\mathrm{CD} 14^{-}, \mathrm{CD}^{-}, \mathrm{CD}^{\mathrm{dim}}\right), \mathrm{CD}^{\mathrm{di}} 6^{+} \mathrm{NK}$ cells $\left(\mathrm{CD} 14^{-}, \mathrm{CD}^{-}, \mathrm{CD} 56^{+}\right)$, naive B cells $\left(\mathrm{CD} 19^{+}, \mathrm{CD} 27^{-}\right)$, memory B cells $\left(\mathrm{CD} 19^{+}, \mathrm{CD}^{2} 7^{+}\right)$, plasmablasts $\left(\mathrm{CD} 19^{+}, \mathrm{CD} 27^{++}\right)$, monocytes $\left(\mathrm{CD} 3^{-}, \mathrm{CD}_{14}{ }^{+}, \mathrm{CD} 11 \mathrm{~b}^{+}\right), \mathrm{CD} 4^{+} \mathrm{T}$ cells $\left(\mathrm{CD}^{+}, \mathrm{CD}^{+}, \mathrm{CD}^{-}\right)$, and $\mathrm{CD} 8^{+} \mathrm{T}$ cells $\left(\mathrm{CD}^{-}, \mathrm{CD}^{+}, \mathrm{CD} 4^{-}\right)$were determined on a MACSQuant Analyzer. CD123 was not used to define pDCs and basophils, due to variable shifts in the expression of this surface marker in the presence of CSL362. Fab'CSL362, the Fab fragment of CSL362, binds to CD123, neutralizing IL-3 signaling, but does not affect ADCC, as it lacks the Fc portion of CSL362. Fab'CSL362 was used to compare the effect of IL-3 blockade alone to that of ADCC on pDCs and basophils. A monocyte gating strategy delineating the 3 major monocyte subsets - classical (CD3 $\left.{ }^{-}, \mathrm{CD}_{14}{ }^{++}, \mathrm{CD} 16^{-}\right)$, nonclassical $\left(\mathrm{CD}^{-}, \mathrm{CD}^{-} 4^{+}, \mathrm{CD} 6^{++}\right)$, and intermediate $\left(\mathrm{CD} 3^{-}, \mathrm{CD} 14^{++}, \mathrm{CD} 16^{+}\right)$- was attempted; however, $\mathrm{CD} 16$ proved an unreliable marker due to shifting expression in the presence of CSL362, presumably due to Fc binding. 
Effect of CSL362 on NK cell activation ex vivo. PBMCs $\left(0.5 \times 10^{6}\right)$ were cultured with $0.01 \mu \mathrm{M}$ CSL362, Fab'CSL362, and two isotype controls for 18 to 21 hours. The first isotype (isotype control) contains the same Fc portion as CSL362, which has been modified to increase affinity for CD16. The second isotype (isotype 2) contains an unmodified IgG1-Fc. All cultures contained a 1:1,000 dilution of GolgiStop to promote intracellular recycling of CD107a. The percentage of viable (Sytox Blue ${ }^{-}$CD107a ${ }^{+}$NK cells $\left(\mathrm{CD}^{-} 4^{-} \mathrm{CD}^{-}, \mathrm{CD}^{-\mathrm{dim}}{ }^{\mathrm{im}}\right.$, and $\left.\mathrm{CD} 56^{+}\right)$was analyzed on a MACSQuant Analyzer.

Effect of CSL362 and anti-IFN- $\alpha$ mAbs on IFN- $\alpha$ production and IFN- $\alpha$-upregulated gene expression ex vivo. PBMCs $\left(0.5 \times 10^{6}\right.$ to $\left.1.0 \times 10^{6}\right)$ were cultured with $0.01 \mu \mathrm{M}$ CSL362, Fab'CSL362, isotype control, or one of two recombinantly produced and purified anti-IFN- $\alpha$ mAbs (KEGG DRUGS database numbers D09668 and D09662) for 6 to 24 hours, before stimulation with either TLR1-9 agonists (Human TLR1-9 Agonist kit, InvivoGen, catalog tlrl-kit1hw) or 50\% sera from SLE patients with low (3.4-7.1 IU/ml), medium (91.8-104.3 IU/ml), or high (>470 IU/ml) anti-dsDNA antibody levels (as measured by radioimmunoassay; normal range $0-4 \mathrm{IU} / \mathrm{ml}$ ) for 18 hours. The TLR agonists were used in the following concentrations - TLR1 (pam3csk4) $1 \mu \mathrm{g} / \mathrm{ml}$, TLR2 (HKLM) $1 \times 10^{8} \mathrm{cells} / \mathrm{ml}$, TLR3 (poly IC) $10 \mu \mathrm{g} / \mathrm{ml}$, TLR4 (LPS) $10 \mu \mathrm{g} / \mathrm{ml}$, TLR5 (flagellin) $2 \mu \mathrm{g} / \mathrm{ml}$, TLR6 (FSL-1) $1 \mu \mathrm{g} / \mathrm{ml}$, TLR7 (imiquimod) $2 \mu \mathrm{g} / \mathrm{ml}$, TLR8 (ssRNA40) $2 \mathrm{~g} / \mathrm{ml}$, and TLR9 (CpG C ODN 2395) $0.5 \mu \mathrm{M}$. When SLE serum was used, fetal calf serum was omitted from culture medium. IFN- $\alpha$ levels in culture supernatants were measured by ELISA. Cell pellets were frozen at $-80^{\circ} \mathrm{C}$ in Qiazol Lysis reagent (Qiagen, catalog 79306) for later RNA extraction.

Effect of depleting $p D C$ s and basophils on IFN- $\alpha$ production ex vivo. PBMCs from healthy and SLE donors were depleted of pDCs (Lin1 ${ }^{-}, \mathrm{HLA}_{-\mathrm{DR}}^{+}, \mathrm{BDCA}^{++}, \mathrm{CD} 123^{++}$) or basophils $\left(\mathrm{Lin}^{-}, \mathrm{CCR} 3^{+}, \mathrm{CD} 123^{++}\right.$) by sorting on a FACSAria. pDC-depleted and basophil-depleted PBMCs $\left(1.0 \times 10^{6}\right)$ were cultured for 18 hours with $0.5 \mu \mathrm{M}$ CpG C (Invivogen, catalog tlrl-2395-1) or $2 \mu \mathrm{g} / \mathrm{ml}$ imiquimod (Invivogen, catalog tlr1imqs). IFN- $\alpha$ levels in culture supernatant were analyzed by ELISA.

Extraction of RNA from PBMCs and whole blood for assessment of IFN- $\alpha$ inducible gene expression. RNA extraction from PBMCs, and from whole blood stored in PAXgene tubes, was performed with the miRNeasy Mini Kit (Qiagen, catalog 217004). The Ambion TURBO DNA-free kit (Invitrogen, catalog AM1907) was used to remove contaminating DNA before RNA was converted to cDNA using the SuperScript III First-Strand Synthesis SuperMix for qRT-PCR kit (Invitrogen, catalog 11752-050). All kits were utilized as per the manufacturers' instructions. Expression of a panel of 11 IFN- $\alpha$-upregulated genes (IFI44L, IFIT1, IFIT3, IRF7, ISG15, MX1, MX2, OAS1, OAS2, SERPING1, XAF1) was determined using TaqMan customized gene arrays (Applied Biosystems, catalog 4342247) analyzed on the 7900HT Fast RT-PCR System (Applied Biosystems).

$R N A$ sequencing of isolated $p D C s$. Healthy donor PBMCs were cultured for 18 hours with $0.5 \mathrm{M} \mathrm{CpG} \mathrm{C,}$ $0.5 \mu \mathrm{g} / \mathrm{ml}$ imiquimod, or media alone for 18 hours. Following culture, cells were stained with Lin1 FITC and magnetically sorted using an Easysep FITC kit (Stemcell Technologies, catalog 18552), keeping only the negative unlabeled fraction. This Lin1- enriched fraction was then stained with Lin1, BDCA1, BDCA2, HLA-DR, CD11c, CD123, and propidium iodide (PI) and sorted using a BD FACS Fusion. pDCs were sorted as $\mathrm{PI}^{-}, \mathrm{Lin}^{-}, \mathrm{HLADR}{ }^{+}, \mathrm{CD} 11 \mathrm{c}^{-}, \mathrm{BDCA}^{+}$, and $\mathrm{CD} 123^{+}$. Sorted pDCs were immediately stored in RNAprotect Cell Reagent (Qiagen, catalog 76526) and frozen at $-80^{\circ} \mathrm{C}$ until RNA extraction using an RNeasy plus micro kit (Qiagen, catalog 74034). RNA was then submitted to the Australian Genome Research Facility for next-generation sequencing on the Illumina HiSeq platform. The data discussed in this publication have been deposited in NCBI's Gene Expression Omnibus (50) and are accessible through GEO Series accession number GSE79272 (http://www.ncbi.nlm.nih.gov/geo/query/acc.cgi?acc=GSE79272).

Effect of CSL362 and anti-IFN- $\alpha$ mAbs on TLR 7 and TLR9 agonist and CD4OL-induced plasmablast expansion and proliferation ex vivo. PBMCs $\left(0.5 \times 10^{6}\right)$ were cultured with $1 \mu \mathrm{g} / \mathrm{ml} \mathrm{CSL362,} \mathrm{one} \mathrm{of} \mathrm{two} \mathrm{recombinantly}$ produced anti-IFN- $\alpha$ mAbs, or isotype control for 24 hours. $0.5 \mu \mathrm{M} \mathrm{CpG} \mathrm{C,} \mathrm{CpG} \mathrm{B/ODN2006} \mathrm{(Invi-}$ voGen, catalog tlrl-2006), CpG A/ODN 2216 (InvivoGen, catalog tlrl-2216), imiquimod, or $0.5 \mu \mathrm{g} / \mathrm{ml}$ CD40L (R\&D Systems, catalog 6245-CL-050) was added for 6 days to stimulate plasmablast expansion. The percentage of viable (Sytox Blue $)$ naive B cells $\left(\mathrm{CD} 19^{+}, \mathrm{CD} 27^{-}\right)$, memory B cells $\left(\mathrm{CD} 19^{+}, \mathrm{CD} 27^{+}\right)$, or plasmablasts $\left(\mathrm{CD} 19^{+}, \mathrm{CD} 27^{++}, \mathrm{CD} 20^{-}, \mathrm{CD}^{2} 8^{++}\right)$was analyzed on a LSR Fortessa. To assess proliferation of the B cell subsets, cells were labeled with CFSE prior to culture for 7 days, as described above.

Effect of reconstituting CSL362-treated PBMCS with pDCs and basophils, or pDC and basophil CM, on TLR7and TLR9-induced plasmablast expansion. pDCs and basophils were isolated from healthy donor PBMCs using the Human Plasmacytoid Dendritic Cell Isolation II Kit (Miltenyi Biotec, catalog 130-097-415) or the 
Human Basophil Kit II (Miltenyi Biotec, catalog 130-092-662), respectively, on an AutoMACS ProSeparator (Miltenyi Biotec). Nondepleted PBMCs $\left(0.5 \times 10^{5}\right)$ were treated with $1 \mu \mathrm{g} / \mathrm{ml} \mathrm{CSL362} \mathrm{or} \mathrm{isotype} \mathrm{for} 24$ hours and then washed 3 times to ensure drug removal. Cultures were reconstituted with isolated pDCs or basophils, at varying concentrations, and stimulated with CpG C, or imiquimod, for 6 days. The percentage of viable plasmablasts was analyzed on a LSR Fortessa.

To produce $\mathrm{CM}$, isolated pDCs and basophils $\left(1.5 \times 10^{5}\right)$ were cultured with $0.5 \mu \mathrm{M} \mathrm{CpG} \mathrm{C}$, imiquimod, or media alone for 24 hours. Supernatants were then added to PBMCs $\left(0.5 \times 10^{5}\right)$ that had been pretreated for 24 hours with $1 \mu \mathrm{g} / \mathrm{ml}$ of CSL362 or isotype control. CpG C or imiquimod or media alone was added to the culture for 6 days. The percentage of viable plasmablasts was analyzed on a LSR Fortessa.

ELISAs. IFN- $\alpha$ levels in supernatant and CM were quantified with the VeriKine Human IFN- $\alpha$ Multisubtype ELISA kit (PBL Assay Science, catalog 41105). BAFF and IL-3 levels in CM were determined by the BAFF Quantikine ELISA kit (R\&D Systems, catalog SBLYSOB) and the IL-3 Duo Set (R\&D Systems, catalog DY203), respectively. Type III IFN levels in supernatant were assessed with the DIY Human IFN lambda 3/1/2 (IL-28B/29/28A) ELISA kit (PBL Assay Science, catalog 61840). Type II IFN levels were determined using the VeriKine Human Interferon Gamma ELISA Kit (PBL Assay Science, catalog 41500-1). All kits were used as per the manufacturers' protocols.

Luminex assays. Levels of 25 cytokines in CM were analyzed using Milliplex Multiplex Assays (Merck Millipore, catalog HT17MG-14K-PX25) on a Luminex 200 analyser. Cytokines analyzed were GM-CSF, IFN- $\gamma$, MIP-3 $\alpha$, TNF- $\alpha$, TNF- $\beta$, IL-1 $\beta$, IL-2, IL-4, IL-5, IL-6, IL-9, IL-10, IL-12p70, IL-13, IL-15, IL-17A, IL-17E/IL25, IL-17F, IL-21, IL-22, IL-23, IL-27, IL-28A, IL-31, and IL-33.

Effect of blockade of IFN- $\alpha, I L-6$, and TNF- $\alpha$ by neutralizing $m A$ bs on plasmablast expansion restored by activated $p D C C M$. PBMCs $\left(0.5 \times 10^{6}\right)$ were cultured with $0.5 \mu \mathrm{M} \mathrm{CpG} \mathrm{C}$ for 6 days, following pretreatment with $1 \mu \mathrm{g} / \mathrm{ml}$ of CSL362 for 24 hours. CM from healthy donor pDCs that had been stimulated with CpG C was added to restore plasmablast expansion at the same time as the $\mathrm{CpG}$, in addition to $50 \mu \mathrm{g} / \mathrm{ml} \mathrm{of}$ a recombinantly produced anti-IFN- $\alpha$ mAb (KEGG Drugs database D09668), an anti-IL-6 mAb (R\&D Systems, catalog MAB2061), or an anti-TNF- $\alpha$ mAb (Etanercept, Pfizer). The percentage of viable plasmablasts was analyzed on a LSR Fortessa.

Effect of s.c. administered CSL362 on pDCs and IFN- $\alpha$-inducible gene expression in cynomolgus macaques. Naive cynomolgus monkeys were administered a single dose $(1 \mathrm{mg} / \mathrm{kg}, 10 \mathrm{mg} / \mathrm{kg}$, or $30 \mathrm{mg} / \mathrm{kg})$ of CSL362 s.c. Peripheral blood was collected at various time points for analysis of CSL362 serum levels by ELISA, and pDC $\left(\mathrm{Lin1}^{-}, \mathrm{HLA}^{-} \mathrm{DR}^{+}, \mathrm{BDCA}^{+}, \mathrm{CD} 123^{+}\right)$and basophil $\left(\mathrm{IgE}^{+}, \mathrm{CD}^{2} 3^{+}\right)$numbers were assessed by flow cytometry on a LSR Fortessa. PBMCs were cultured with CpG C for 24 hours, after which expression of a panel of IFN- $\alpha$-inducible genes (IFI35, IFIT1, IRF7, MX1, MX2, OAS1) was determined by quantitative PCR.

Statistics. Comparisons between two groups were analyzed with the Mann Whitney $U$ test. A $P$ value of less than 0.05 was considered statistically significant. Statistical analyses were performed with GraphPad Prism Software (version 6.0).

Study approval. Animal studies were conducted at Maccine Pte Ltd, Singapore, in accordance with standard operating procedures and were approved by the Institutional Animal Care and Use Committee of Maccine (259-2012, amendment 34). The human research ethics committees of Melbourne Health (2012.039) and The Walter and Eliza Hall Institute of Medical Research (12/05) in Parkville, approved the human studies. All human subjects signed a written consent form prior to participation in the study.

\section{Author contributions}

SO, GV, EM, ADN, IPW, and NJW designed the research. SO, HH, TYT, MN, MB, and KM performed the experiments. SO, MN, KM, MB, IPW, and NJW analyzed the data. SO, IPW, and NJW wrote the manuscript. All authors assisted in revising the manuscript.

\section{Acknowledgments}

We thank Jenni Harris, Lina Laskos, Naomi Sprigg, and Cathy Quillici at The Walter and Eliza Hall Institute's Volunteer Blood Donor Registry and Susan Tadros, Clare O'Neill, and our clinical colleagues in the rheumatology, renal, and dermatology units at The Royal Melbourne Hospital for their assistance with donor recruitment. We thank the donors for contributing samples to this study. RNA sequencing was performed by the Australian Genome Research Facility. This work was supported by the Reid Charitable Trusts, the National Health and Medical Research Council (NHMRC) Australia (I.P. Wicks Clinical 
Practitioner Fellowship [1023407], NHMRC Program Grant ([1016647], S. Oon Postgraduate Scholarship [1039026]), operational infrastructure grants through the Australian government's Institute for Research and Innovation in Social Services, the Victorian State Government, Arthritis Australia (South Australia Lupus, Scleroderma, and Sjogren's Support Group grant), CSL Limited, and Janssen Research and Development.

Address correspondence to: Ian P. Wicks, Division of Inflammation, The Walter and Eliza Hall Institute of Medical Research, 1G Royal Parade, Parkville, Victoria, Australia, 3052. Phone: 61.3.9345.2466; E-mail: wicks@wehi.edu.au. Or to: Nicholas Wilson, CSL Limited, Bio21 Institute, 30 Flemington Road, Parkville, Victoria, Australia, 3052. Phone: 61.3.9389.2153; E-mail: nick.wilson@csl.com.au.

Tsin Yee Tai's present address is: Division of Inflammation, The Walter and Eliza Hall Institute of Medical Research, Parkville, Victoria, Australia.

1. Bernatsky S, et al. Mortality in systemic lupus erythematosus. Arthritis Rheum. 2006;54(8):2550-2557.

2. Borchers AT, Keen CL, Shoenfeld Y, Gershwin ME. Surviving the butterfly and the wolf: mortality trends in systemic lupus erythematosus. Autoimmun Rev. 2004;3(6):423-453.

3. Bennett L, et al. Interferon and granulopoiesis signatures in systemic lupus erythematosus blood. J Exp Med. 2003;197(6):711-723.

4. Bauer JW, et al. Elevated serum levels of interferon-regulated chemokines are biomarkers for active human systemic lupus erythematosus. PLoS Med. 2006;3(12):2274-2284.

5. Petri M, et al. Sifalimumab, a human anti-interferon-alpha monoclonal antibody, in systemic lupus erythematosus: a phase I randomized, controlled, dose-escalation study. Arthritis Rheum. 2013;65(4):1011-1021.

6. McBride JM, et al. Safety and pharmacodynamics of rontalizumab in patients with systemic lupus erythematosus: results of a phase I, placebo-controlled, double-blind, dose-escalation study. Arthritis Rheum. 2012;64(11):3666-3676.

7. Kalunian K, et al. A phase II study of the efficacy and safety of rontalizumab (rhuMAb inteferon- $\alpha$ ) in patients with systemic lupus erythematosus (ROSE). Ann Rheum Dis. 2016;75(1):196-202.

8. Furie R, et al. Anifrolumab, an anti-interferon alpha receptor monoclonal antibody, in moderate to severe systemic lupus erythematosus (SLE). Arthritis Rheumatol. 2015; 67(suppl 10):3223.

9. Liu YJ. IPC: professional type 1 interferon-producing cells and plasmacytoid dendritic cell precursors. Annu Rev Immunol. 2005;23:275-306.

10. Llanos C, Mackern-Oberti JP, Vega F, Jacobelli SH, Kalergis AM. Tolerogenic dendritic cells as a therapy for treating lupus. Clin Immunol. 2013;148(2):237-245.

11. Banchereau J, Pascual V. Type I interferon in systemic lupus erythematosus and other autoimmune diseases. Immunity. 2006;25(3):383-392

12. Båve U, Magnusson M, Eloranta ML, Perers A, Alm GV, Rönnblom L. Fc $\gamma$ RIIa is expressed on natural IFN- $\alpha$-producing cells (plasmacytoid dendritic cells) and is required for the IFN- $\alpha$ production induced by apoptotic cells combined with lupus IgG. J Immunol. 2003;171(6):3296-3302.

13. Boule MW, Broughton C, Mackay F, Akira S, Marshak-Rothstein A, Rifkin IR. Toll-like receptor 9-dependent and -independent dendritic cell activation by chromatin-immunoglobulin G complexes. J Exp Med. 2004;199(12):1631-1640.

14. Honda K, et al. IRF-7 is the master regulator of type-I inteferon-dependent immune responses. Nature. 2005;434(7034):772-777.

15. Means TK, Latz E, Hayashi F, Murali MR, Golenbock DT, Luster AD. Human lupus autoantibody-DNA complexes activate DCs through cooperation of CD32 and TLR9. J Clin Invest. 2005;115(2):407-417.

16. Chan VS, Nie YJ, Shen N, Yan S, Mok MY, Lau CS. Distinct roles of myeloid and plasmacytoid dendritic cells in systemic lupus erythematosus. Autoimmun Rev. 2012;11(12):890-897.

17. Sisirak V, et al. Genetic evidence for the role of plasmacytoid dendritic cells in systemic lupus erythematosus. $J$ Exp Med 2014;211(10):1969-1976.

18. Rowland SL, et al. Early, transient depletion of plasmacytoid dendritic cells ameliorates autoimmunity in a lupus model. $J$ Exp Med. 2014;211(10):1977-1991.

19. Farkas L, Beiske K, Lund-Johansen F, Brandtzaeg P, Jahnsen F. Plasmactyoid dendritic cells (natural interferon- $\alpha / \beta$-producing cells) accumulate in cutaneous lupus erythematous lesions. Am J Pathol. 2001;159(1):237-243.

20. Cederblad B, Blomberg S, Vallin H, Perers A, Alm GV, Rönnblom L. Patients with systemic lupus erythematosus have reduced numbers of circulating natural interferon- $\alpha$ producing cells. J Autoimmun. 1998;11(5):465-470.

21. Blomberg S, Eloranta ML, Magnusson M, Alm GV, Rönnblom L. Expression of the markers BDCA-2 and BDCA-4 and production of interferon- $\alpha$ by plasmacytoid dendritic cells in systemic lupus erythematosus. Arthritis Rheum. 2003;48(9):2524-2532.

22. Jin $\mathrm{O}$, et al. Systemic lupus erythematosus patients have increased number of circulating plasmacytoid dendritic cells, but decreased myeloid dendritic cells with deficient CD83 expression. Lupus. 2008;17(7):654-662.

23. Miyashita A, et al. Proportion of lymphocytic inflammation with CD123 positive cells in lupus erythematous profundus predict a clinical response to treatment. Acta Derm Venereol. 2014;94(5):563-567.

24. Lande R, et al. 2011. Neutrophils activate plasmacytoid dendritic cells by releasing self-DNA-peptide complexes in systemic lupus erythematosus. Sci Transl Med. 2011;3(73):73ra19.

25. Dzionek A, et al. BDCA-2, a novel plasmacytoid dendritic cell-specific type II C-type lectin, mediates antigen capture and is a potent inhibitor of interferon $\alpha / \beta$ induction. $J$ Exp Med. 2001;194(12):1823-1834.

26. Pellerin A, et al. Anti-BDCA2 monoclonal antibody inhibits plasmacytoid dendritic cell activation through Fc-dependent and Fc-independent mechanisms. EMBO Mol Med. 2015;7(4):464-476. 
27. Zhan Y, et al. Bcl-2 antagonists kill plasmacytoid dendritic cells from lupus-prone mice and dampen interferon-alpha production. Arthritis Rheumatol. 2015;67(3):797-808.

28. Olweus J, et al. Dendritic cell ontogeny: a human dendritic cell lineage of myeloid origin. Proc Natl Acad Sci U S A. 1997;94(23):12551-12556.

29. Broughton SE, et al. Dual mechanism of interleukin-3 receptor blockade by an anti-cancer antibody. Cell Rep. 2014;8(2):410-419.

30. Busfield SJ, et al. Targeting of acute myeloid leukemia in vitro and in vivo with an anti-CD123 mAb engineered for optimal ADCC. Leukemia. 2014;28(3):2213-2221.

31. Charles N, Hardwick D, Daugas E, Illei GG, Rivera J. Basophils and the T helper 2 environment can promote the development of lupus nephritis. Nat Med. 2010;16(6):701-707.

32. Grouard G, Rissoan M, Filgueira L, Durand I, Banchereau J, Liu YJ. The enigmatic plasmacytoid T cells develop into dendritic cells with interleukin (IL)-3 and CD40-ligand. J Exp Med. 1997;185(6):1101-1111.

33. Kadowaki N, et al. Subsets of human dendritic cell precursors express different toll-like receptors and respond to different microbial antigens. J Exp Med. 2001;194(6):863-869.

34. Jego G, Palucka AK, Blanck JP, Chalouni C, Pascual V, Banchereau J. Plasmacytoid dendritic cells induce plasma cell differentiation through type I interferon and interleukin 6. Immunity. 2003;19(2):225-234.

35. Hervier B, et al. Phenotype and function of natural killer cells in systemic lupus erythematosus: excess interferon-gamma production in patients with active disease. Arthritis Rheum. 2011;63(6):1698-1706.

36. Lin SC, Kuo CC, Tsao JT, Lin LJ. Profiling the expression of interleukin (IL)-28 and IL-28 receptor $\alpha$ in systemic lupus erythematosus patients. Eur J Clin Invest. 2012;42(1):61-69.

37. Wu Q, Yang Q, Lourenco E, Sun H, Zhang Y. Interferon-lambda1 induces peripheral blood mononuclear cell-derived chemokines secretion in patients with systemic lupus erythematosus: its correlation with disease activity. Arthritis Res Ther. 2011;13(3):R88

38. Amezcua-Guerra LM, Ferrusquia-Toriz D, Castillo-Martínez D, Márquez-Velasco R, Chávez-Rueda AK, Bojalil R. Limited effectiveness for the therapeutic blockade of interferon $\alpha$ in systemic lupus erythematosus: a possible role for type III interferons. Rheumatology (Oxford). 2015;54(2):203-205.

39. Davidson A, Diamond B. Activated basophils give lupus a booster shot. Nat Med. 2010;16(6):635-636.

40. Voehringer D. Basophil modulation by cytokine instruction. Eur J Immunol. 2012;42(10):2544-2550.

41. Fishman P, et al. Interleukin-3 immunoassay in systemic lupus erythematosus: preliminary data. Int Arch Allergy Immunol. $1993 ; 100(3): 215-218$

42. Renner K, et al. IL-3 contributes to development of lupus nephritis in MRL/lpr mice. Kidney Int. 2015;88(5):1088-1098.

43. Smith BD, et al. 120 first-in man, phase I study of CSL362 (anti-IL3R $\alpha$ /anti-CD123 monoclonal antibody) in patients with $\mathrm{CD}_{123^{+}}$acute myeloid leukemia (AML) in CR at high risk for early relapse. Blood. 2014;124(21):120.

44. Cao W. Pivotal functions of plasmacytoid dendritic cells in systemic autoimmune pathogenesis. J Clin Cell Immunol. 2014;5(2):212-234.

45. Rodríguez-Carrio J, López P, Suárez A. Type I IFNs as biomarkers in rheumatoid arthritis: towards disease profiling and personalized medicine. Clin Sci (Lond). 2015;128(8):449-464.

46. Goldberg A, et al. Dose-escalation of human anti-interferon-alpha receptor monoclonal antibody MEDI-546 in subjects with systemic sclerosis: a phase 1, multicenter, open label study. Arthritis Res Ther. 2014;16(1):R57.

47. Bissonnette R, et al. A randomized, double-blind, placebo-controlled, phase I study of MEDI-545, an anti-interferon-alpha monoclonal antibody, in subjects with chronic psoriasis. J Am Acad Dermatol. 2010;62(3):427-436.

48. American College of Rheumatology Ad Hoc Committee on Systemic Lupus Erythematosus Response Criteria. The American College of Rheumatology response criteria for systemic lupus erythematosus clinical trials: measures of overall disease activity. Arthritis Rheum. 2004;50(11):3418-3426.

49. Petri M, et al. Derivation and validation of the Systemic Lupus International Collaborating Clinics classification criteria for systemic lupus erythematosus. Arthritis Rheum. 2012;64(8):2677-2686.

50. Edgar R, Domrachev M, Lasha AE. Gene expression omnibus: NCBI gene expression and hybridization array data repository. Nucleic Acids Res. 2002;30(1):207-210. 